\title{
27. EARLY PALEOGENE BENTHIC FORAMINIFERAL ASSEMBLAGES AND STABLE ISOTOPES IN THE SOUTHERN OCEAN1
}

\author{
Miriam E. Katz ${ }^{2}$ and Kenneth G. Miller ${ }^{3}$
}

\begin{abstract}
ODP Leg 114 recovered sections at four sites east of the Falkland Plateau that cover a wide range of paleodepths and provide the opportunity to evaluate the response of benthic foraminifers to late Paleocene and Eocene oceanographic changes. Early Paleogene paleodepth estimates were obtained by "backtracking" assuming simple thermal subsidence (Site 698, $\sim 900 \mathrm{~m}$; Site 702, $2000 \mathrm{~m}$; Site 700, $\sim 2400 \mathrm{~m}$; and Site 699, $\sim 2800 \mathrm{~m}$ ). These estimates agree with paleodepths determined by comparing our quantitative benthic foraminiferal assemblages to previously published assemblages associated with known paleodepths.

Previous studies document that a major benthic foraminiferal crisis occurred in the latest Paleocene in the Atlantic, Caribbean, and Pacific; a similar faunal turnover occurred in the latest Paleocene throughout the Atlantic sector of the Southern Ocean. At the Leg 114 sites, Stensioina beccariiformis-dominated assemblages were replaced by Nuttallides truempyi-dominated assemblages just prior to the Paleocene/Eocene boundary. A preponderance of benthic foraminiferal taxa last appeared immediately prior to the Paleocene/Eocene boundary, as recognized at these high latitudes by the last appearance of the calcareous nannofossil Fasciculithus spp. and the first appearance of the planktonic foraminifer Pseudohastigerina spp. Recovery and biostratigraphic control at the Leg 114 sites is insufficient to constrain precisely the timing of the extinction event, although studies of material from the Maud Rise (Weddell Sea) suggest that it occurred in the latest Paleocene.

The benthic foraminiferal crisis may have been caused by deep-water warming, a drop in food supply, or changing deep-water source regions. Oxygen isotope data show that there is no clear correlation between $\delta^{18} \mathrm{O}$ changes and extinctions. Similarly, most of the extinctions occurred well after the start of the drop in global $\delta^{13} \mathrm{C}$ values, which may, in part, reflect a decrease in productivity. Interbasinal carbon isotope comparisons suggest that the Southern Ocean was supplied "young" (high $\mathrm{O}_{2}$, low nutrient, and high $\delta^{13} \mathrm{C}$ ) deep water in the latest Paleocene (approximately 60-58 Ma) and early Eocene (approximately 57-52 Ma). Oxygen isotope evidence indicates that the Southern Ocean was filled with cooler water than that in the Pacific beginning at approximately $60 \mathrm{Ma}$, supporting our contention that the deep-water source was antarctic. However, near the Paleocene/Eocene boundary (approximately 58-57 Ma), the supply of Southern Ocean "young" deep water was reduced or eliminated. We speculate that elimination of this inferred antarctic source between 58 and $57 \mathrm{Ma}$ triggered the benthic foraminiferal turnover.
\end{abstract}

\section{BACKGROUND}

\section{Faunal Changes}

One of the most important deep-sea benthic foraminiferal faunal turnovers of the Cenozoic occurred near the end of the Paleocene (Berggren and Miller, in press) and was accompanied by major oxygen and carbon isotope excursions. The extinction event has been documented in the Atlantic (Tjalsma, 1976; Schnitker, 1979; Tjalsma and Lohmann, 1983) and Pacific (Miller et al., 1987c) oceans. Similar late Paleocene benthic foraminiferal faunal changes were noted in the north-

Editor's note: The stratigraphic framework used herein is that of Ciesielski, Kristoffersen, et al. (1988), which is based largely upon shipboard data. Insufficient time was available for revision incorporating the more detailed biostratigraphic-magnetostratigraphic results presented elsewhere in this volume. These new data do not adversely change the conclusions and ages presented here, which describe largely long-term changes in Paleogene benthic foraminifer assemblages and paleoenvironment. Where appropriate, the editor (PFC) has inserted references to important stratigraphic datums based upon the biostratigraphic-magnetostratigraphic synthesis (P. F. Ciesielski, unpubl. data) of Leg 114.

${ }^{1}$ Ciesielski, P. F., Kristoffersen, Y., et al., 1991. Proc. ODP, Sci. Results, 114: College Station, TX (Ocean Drilling Program).

2 Lamont-Doherty Geological Observatory of Columbia University, Palisades, NY 10964.

${ }^{3}$ Department of Geological Science, Rutgers University, New Brunswick, NJ 08903, and Lamont-Doherty Geological Observatory of Columbia University, Palisades, NY 10964. ern Italian Pessagno section (Braga et al., 1975) and in the Indian Ocean (Shipboard Scientific Party, 1974; Vincent et al., 1974). Fifty percent of the Paleocene benthic foraminiferal taxa last appeared between samples in Zones P5 and P6a from Atlantic and Caribbean locations (Tjalsma and Lohmann, 1983). At Pacific Ocean Deep Sea Drilling Project (DSDP) Site 577 , more than $50 \%$ of the benthic foraminiferal species disappeared between planktonic foraminiferal Zones P4 and P6b (Miller et al., 1987c). This turnover culminated at Site 577 between a sample assigned to the uppermost Paleocene Zone P6a and a sample assigned to the lowermost Eocene Zone P6b. Still, the precise timing of this extinction event (either latest Paleocene or earliest Eocene) remains unclear in the Atlantic and Pacific oceans. In addition, the extent of the faunal turnover has not been established in other basins, nor has it been established over a depth transect representing a wide bathymetric range. Ocean Drilling Program (ODP) Legs 113 and 114 recovered Southern Ocean sections spanning the Paleocene/Eocene boundary, which allows evaluation of this taxonomic turnover across a wide paleodepth range (Katz and Miller, 1988, this study; Thomas, 1988, 1990, in press).

Schnitker (1979) and Tjalsma and Lohmann (1983) noted that the bathyal to upper abyssal faunas were most severely impacted by the extinction event near the end of the $\mathrm{Pa}$ leocene. Tjalsma and Lohmann (1983) noted that this fauna consisted of relict Cretaceous taxa of the presumably thermophilic Stensioina beccariiformis assemblage. Thomas (1988, 1990, in press) documented that relict Cretaceous benthic 
foraminifers became extinct in the latest Paleocene at the Antarctic Maud Rise Sites 689 and 690 in the Weddell Sea, with a $50 \%$ decrease in diversity within $50,000 \mathrm{yr}$. This firmly establishes the timing of the extinction in the Southern Ocean, but the cause of the event is still unknown.

\section{Stable Isotope Changes}

The cause(s) of the late Paleocene extinctions has remained elusive, in part because of the lack of supporting paleoenvironmental data. Little information on bottom-water temperature was available for this interval from the pioneering studies of Savin et al. (1975) or Shackleton and Kennett (1975). As a result, Schnitker (1979) and Tjalsma and Lohmann (1983) found no relationship between temperature and the Paleocene/ Eocene faunal turnover. Improved recovery of PaleoceneEocene sections provided the requisite material for showing that a major bottom-water warming occurred during the latest Paleocene to earliest Eocene (Shackleton et al., 1984; Miller et al., 1987a, 1987c), and we have speculated that it was this warming that was primarily responsible for the benthic foraminiferal faunal turnover (Miller et al., 1987c; see also Thomas, 1988, 1990, in press). In addition, two other potential causes may have been important: an inferred drop in surface ocean productivity (hence, food supply) near the Paleocene/ Eocene boundary (Shackleton et al., 1985b) and a change in deep-water source regions (Miller et al., 1987c; Thomas, 1988, 1990 , in press). In order to test possible causes, it is critical that stable isotope data be obtained from the same levels examined for benthic foraminifers.

Previous studies of early Paleogene benthic foraminiferal oxygen and carbon isotopes have focused on the South Atlantic (Shackleton and Hall, 1984; Shackleton et al., 1984; Oberhansli et al., 1984; Oberhansli and Toumarkine, 1985) and Pacific oceans (Savin et al., 1975; Miller et al., 1987c). Although early Paleogene isotope data are available from the North Atlantic (Paleocene-Boersma et al., 1979; Miller et al., 1987c; Eocene-Vergnaud-Grazzini et al., 1978; Miller and Curry, 1982; Miller et al., 1985), there are no North Atlantic data available spanning the critical latest Paleocene to early Eocene interval. The previous record of the Southern Ocean is similarly limited: the only data available prior to Legs 113 and 114 were latest Paleocene to Eocene records from the Campbell Plateau (Shackleton and Kennett, 1975) and Eocene records from the Falkland Plateau and southeast Argentine Basin (Muza et al., 1983).

All locations examined show a distinct decrease in $\delta^{18} \mathrm{O}$ values from the latest Paleocene to earliest Eocene; estimates of the warming range from $3^{\circ}$ to $6^{\circ} \mathrm{C}$ (Miller et al., 1987c). The lowest $\delta^{18} \mathrm{O}$ values of the Cenozoic were attained in the early Eocene at intermediate, deep, and bottom locations in every ocean basin examined. Oxygen isotope values began a general increase from the latest early Eocene through the Oligocene, occurring as three distinct steps:

1. An increase of approximately $1.0 \%$ oo began near the early/middle Eocene boundary (approximately 52-51 Ma; Shackleton et al., 1984; Oberhansli et al., 1984; Oberhansli and Toumarkine, 1985; Miller et al., 1987c, 1987a; time scale of Berggren et al., 1985). The exact timing of this increase was uncertain until this study.

2. An increase of $0.5 \%-1.0 \%$ occurred near the middle/ late Eocene boundary (Keigwin and Corliss, 1986; Oberhansli et al., 1984; Oberhansli and Toumarkine, 1985; Miller et al., 1987a). The exact timing of this increase remains poorly known.

3. An increase of $1.0 \%-1.5 \%$ oo occurred in the earliest Oligocene (approximately $35.8 \mathrm{Ma}$; age estimate derived from magnetochronology at Site 522; Miller et al., 1988).
This well-known $\delta^{18} \mathrm{O}$ increase (e.g., Vergnaud-Grazzini and Oberhansli, 1986; Keigwin and Corliss, 1986) signaled the first definite evidence of glacial ice sheets on Antarctica (Miller et al., 1987a). The cause of the Eocene $\delta^{18} \mathrm{O}$ increases has generally been ascribed to deep-water cooling (e.g., Savin et al., 1975; Shackleton and Kennett, 1975; Miller et al., 1988). However, a major issue remains: it is possible that ice sheets developed prior to the Oligocene (e.g., Barron et al., 1988). We address the first two of the Eocene $\delta^{18} \mathrm{O}$ increases in this contribution.

Large changes in the $\delta^{13} \mathrm{C}$ budget occurred during the late Paleocene to early Eocene (Shackleton and Hall, 1984; Shackleton et al., 1985a, 1985b; Oberhansli et al., 1984; Oberhansli and Toumarkine, 1985; Hsü et al., 1985). The cause of high $\delta^{13} \mathrm{C}$ values and strong surface-water $\delta^{13} \mathrm{C}$ gradients in the late Paleocene Pacific and Atlantic oceans (Boersma and Premoli Silva, 1983; Shackleton et al., 1985a, 1985b) remains controversial; the high late Paleocene $\delta^{13} \mathrm{C}$ values have been suggested as indicating high global productivity (Shackleton and Hall, 1984), high accumulation rates of sedimentary organic carbon (Shackleton, 1987), and an enhanced oxygen-minimum zone (Shackleton et al., 1985a). The large decrease in $\delta^{13} \mathrm{C}$ values across the Paleocene/Eocene boundary was inferred to represent a global drop in surface ocean productivity (Shackleton et al., 1985b). Miller et al. (1987c) noted that there was no change in the vertical $\delta^{13} \mathrm{C}$ gradient between benthic and planktonic foraminiferal values across the Paleocene/Eocene boundary, which weakens the case for a change in ocean productivity (see discussions in Miller et al., 1987c; Thomas, 1990).

Several key early Paleogene oceanographic problems can be addressed by obtaining more complete stable isotope records from the Southern Ocean. Perhaps the most intriguing problem related to the benthic foraminiferal turnover issue concerns the potential sources of early Paleogene deep water. The warm deep-water conditions $\left(10^{\circ} \mathrm{C}\right)$ of the Cretaceous and early Paleogene have been inferred as reflecting production of warm saline bottom water at low latitudes (Brass et al., 1982). In contrast, Barrera et al. (1987) suggested that Late Cretaceous to Paleocene bottom water was produced near the poles as today because surface-water $\delta^{18} \mathrm{O}$ values near Antarctica were similar to those found in the deep sea at lower latitudes. Miller et al. (1987c) have shown that the Southern Ocean was enriched in ${ }^{13} \mathrm{C}$ relative to the Pacific and North Atlantic oceans during the late Paleocene, indicating that the Southern Oceans were proximal to a "young" (nutrient depleted, oxygen-rich) deep-water source. We infer this to have been a Southern Ocean source as present today. Oxygen isotope data from the Maud Rise have been used to suggest that a lowlatitude source of warm saline deep water entered the Southern Ocean during the early to middle Eocene (Kennett and Stott, 1988). Our previous late Paleocene reconstructions clearly suggest that the Southern Ocean was close to its source of deep water. However, our early Eocene carbon isotope comparisons were equivocal (based upon only two Southern Ocean points), and no data were available for the middle to late Eocene (Miller et al., 1987c).

ODP Leg 114 drilled four sites east of the Falkland Plateau at which Paleocene and Eocene sections were recovered (Figs. 1 and 2). These sites cover a wide present depth range (Site 698, 2128 m; Site 699, 3707 m; Site 700, 3598 m; and Site $702,3084 \mathrm{~m}$ ) and provide a good paleobathymetric transect of the benthic foraminiferal faunal data and of the oxygen and carbon isotope records. These four Leg 114 sites provide us with a unique opportunity to study the late Paleocene benthic foraminiferal faunal turnover across a depth transect representing a wide paleobathymetric range (Fig. 3). This transect 


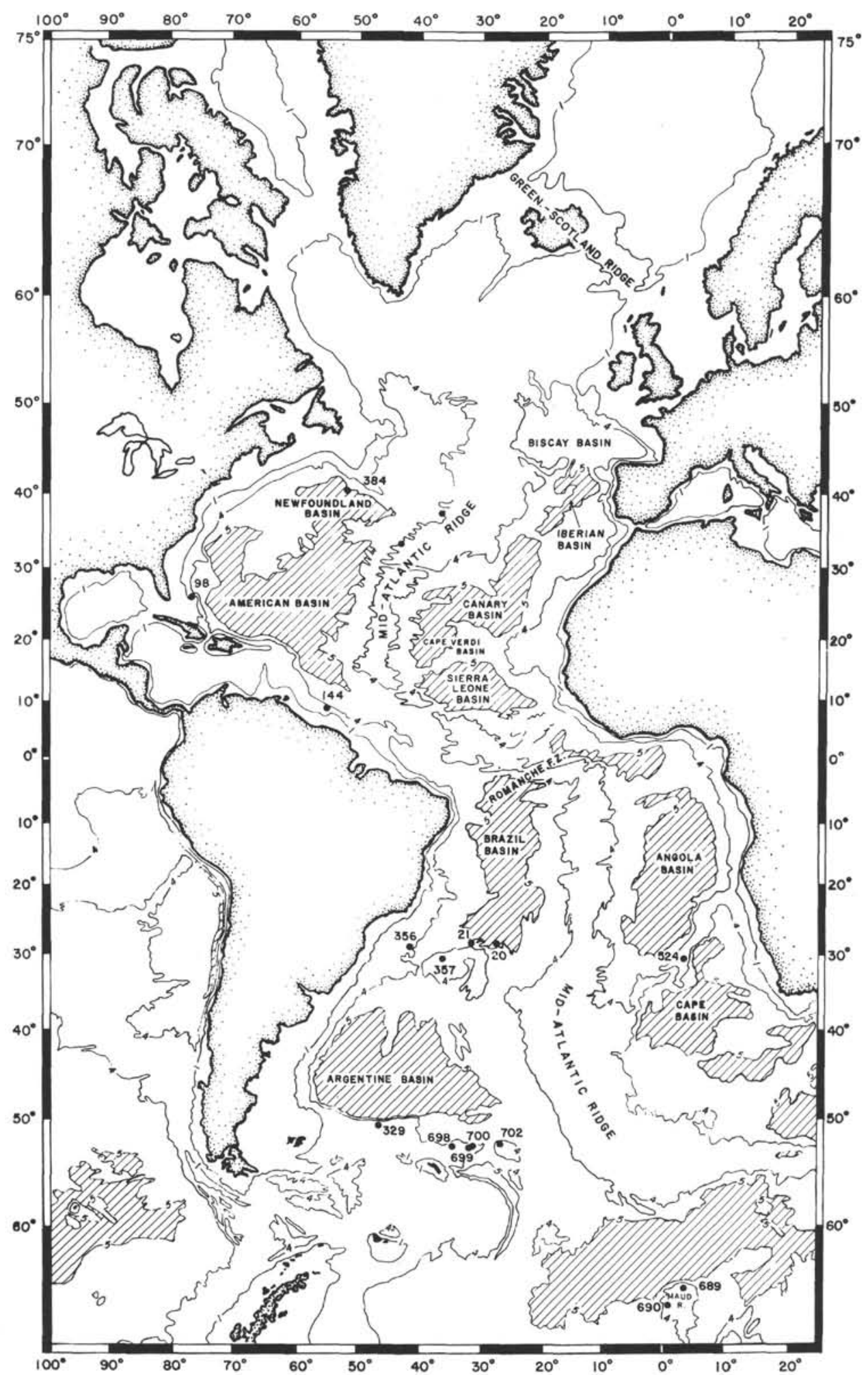

Figure 1. Bathymetric location map showing sites mentioned in text; the 1-, 4-, and 5-km contour intervals are indicated, and depths greater than $5 \mathrm{~km}$ are crosshatched. F.Z. = Fracture Zone. Modified after Deep Sea Drilling Project (unpubl. data). 


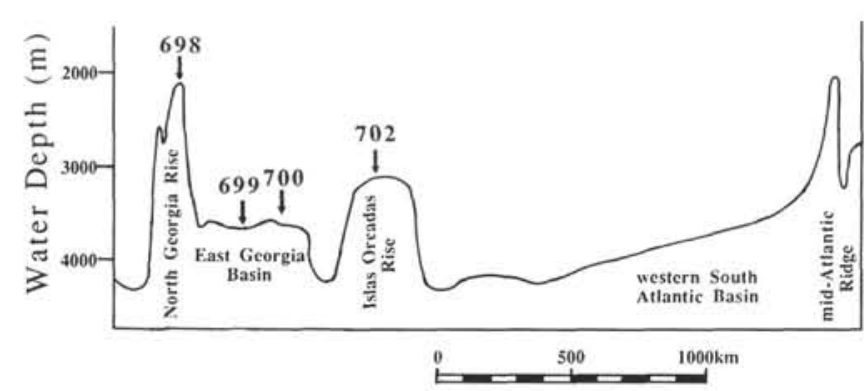

Figure 2. Schematic bathymetric cross section of the western South Atlantic Basin showing the present depths of Leg 114 Sites 698, 699, 700 , and 702 .

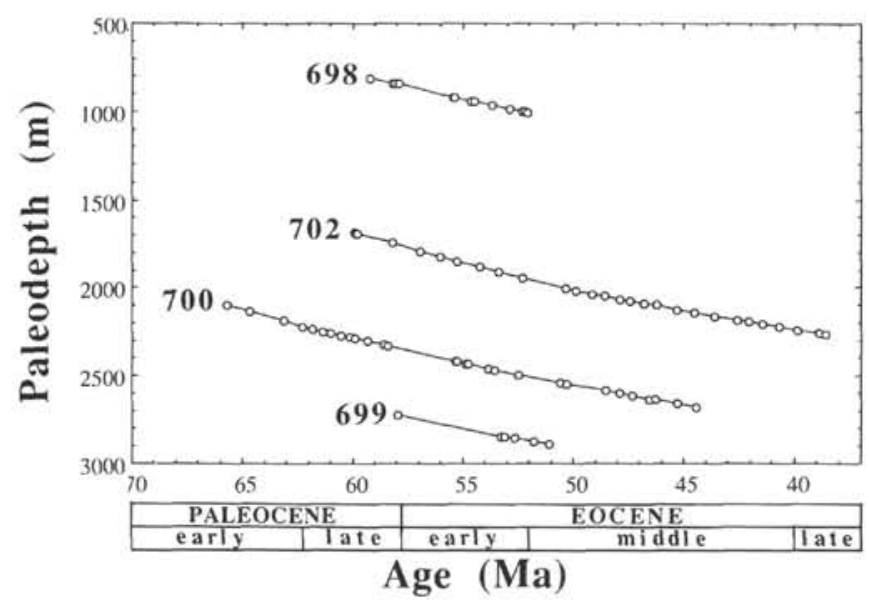

Figure 3. Paleodepth vs, age transect of samples from Leg 114 Sites $698,699,700$, and 702 , which were examined for quantitative benthic foraminiferal analyses. Parameters are as follows: Site 698, present water depth of $2128 \mathrm{~m}$, sediment thickness of $210 \mathrm{~m}$, basement age of $90 \mathrm{Ma}$, and pre-exponential empirical constant of -3650 ; Site 699, present water depth of $3707.5 \mathrm{~m}$, sediment thickness of $700 \mathrm{~m}$, basement age of $80 \mathrm{Ma}$, and pre-exponential empirical constant of -3650 ; Site 700 , present water depth of $3598 \mathrm{~m}$, sediment thickness of $583 \mathrm{~m}$, basement age of $87 \mathrm{Ma}$, and pre-exponential empirical constant of -3650 ; and Site 702, present water depth of $3083 \mathrm{~m}$, sediment thickness of $400 \mathrm{~m}$, basement age of $70 \mathrm{Ma}$, and empirical constant of 300 .

allows us to evaluate depth-related influences on the extinction event.

\section{METHODS}

Paleocene and Eocene sections were studied from four Leg 114 drill sites: Hole $698 \mathrm{~A}\left(51^{\circ} 27.51^{\prime} \mathrm{S}, 33^{\circ} 05.96^{\prime} \mathrm{W}\right)$; Hole $699 \mathrm{~A}$ $\left(51^{\circ} 32.537^{\prime} \mathrm{S}, \quad 30^{\circ} 40.619^{\prime} \mathrm{W}\right)$; Hole $700 \mathrm{~B} \quad\left(51^{\circ} 31.977^{\prime} \mathrm{S}\right.$, $\left.30^{\circ} 16.688^{\prime} \mathrm{W}\right)$; and Hole $702 \mathrm{~B}\left(50^{\circ} 56.786^{\prime} \mathrm{S}, 26^{\circ} 22.117^{\prime} \mathrm{W}\right)$ (Fig. 1). Samples were obtained aboard ship at approximately one per section $(1.5 \mathrm{~m})$. This corresponds to a typical sampling interval of 0.5 to $1.0 \mathrm{~m} . \mathrm{y}$. for the Paleocene-Eocene sections where recovery is adequate.

Age estimates were based upon biostratigraphic and magnetostratigraphic correlations. We used the revised shipboard biostratigraphic datum levels, emphasizing nannofossil zonal boundaries and chronozones along with planktonic foraminiferal datum levels (Ciesielski, Kristoffersen, et al., 1988) (Table 1). The time scale of Berggren et al. (1985) was used for the ages of the zonal and stratigraphic boundaries, and the ages of
Table 1. Age model parameters, Holes 698A, 699A, 700B, and $702 B$.

\begin{tabular}{|c|c|c|}
\hline Level $^{\mathrm{a}}$ & $\begin{array}{l}\text { Age } \\
\text { (Ma) }\end{array}$ & $\begin{array}{l}\text { Depth } \\
\text { (mbsf) }\end{array}$ \\
\hline \multicolumn{3}{|l|}{ Hole 698A } \\
\hline $\begin{array}{l}\text { Level within range Morozovella caucasica }(= \\
\text { M. crater) }\end{array}$ & $>52$ & 4.00 \\
\hline LO Fasciculithus & 57.4 & 66.91 \\
\hline Base NP9 & 59.2 & 81.26 \\
\hline \multicolumn{3}{|l|}{ Hole 699A } \\
\hline Base NP15 & 49.8 & 416.25 \\
\hline Base NP14 & 52.6 & 454.8 \\
\hline Top NP12 & 53.7 & 472.85 \\
\hline LO Fasciculithus & 57.4 & 493.38 \\
\hline Base NP9 & 59.2 & 508.83 \\
\hline \multicolumn{3}{|l|}{ Hole 700B } \\
\hline FO Globogerinatheka index & 45.0 & 44.50 \\
\hline LO Tribrachiatus orthostylus & 53.7 & 172.30 \\
\hline LAD Fasciculithus & 57.4 & 228.80 \\
\hline Base NP9 (= FO Discoaster multiradiatus) & 59.2 & 245.60 \\
\hline Base NP5 (= FO Fasciculithus tympaniformis) & 62.0 & 293.90 \\
\hline Chron C26R/C27N & 63.03 & 299.15 \\
\hline Chron C28R/C29N & 65.5 & 327.20 \\
\hline \multicolumn{3}{|l|}{ Hole $702 \mathrm{~B}$} \\
\hline Extrapolation of sedimentation rate & 39.19 & $\sim 29$ \\
\hline LO Acarinina primitiva & 40.6 & 46.05 \\
\hline Base C18N & 42.73 & 71.90 \\
\hline Top C19N & 43.60 & 84.26 \\
\hline Base C19N & 44.06 & 86.50 \\
\hline Top $\mathrm{C} 20 \mathrm{~N}$ & 44.66 & 98.70 \\
\hline Base C20N & 46.17 & 113.40 \\
\hline Top C21N & 48.75 & 157.30 \\
\hline Base $\mathrm{C} 21 \mathrm{~N}$ & 50.34 & 180.30 \\
\hline$\sim$ & 50.408 & 181.29 \\
\hline Top C22N? & 51.95 & 181.30 \\
\hline Base C22N & 52.62 & 193.50 \\
\hline Top NP12 & 53.70 & 200.23 \\
\hline LAD Fasciculithus & 57.4 & 239.63 \\
\hline Base NP9 & 59.2 & 249.38 \\
\hline Base NP8 & 59.9 & 276.58 \\
\hline
\end{tabular}

${ }^{a} \mathrm{LO}=$ last occurrence $; \mathrm{FO}=$ first occurrence LAD $=$ last-appearance datum.

our samples were established by linearly interpolating between datum levels and chronozonal boundaries (Table 1).

Calibrations of high-latitude sections with lower latitude sections and with the stratotypes are uncertain. Berggren et al. (1985) recognized the Paleocene/Eocene boundary at low latitudes based on the last occurrence of the planktonic foraminifer Morozovella velascoensis (top of Zone P6a) and assigned an age estimate of 57.8 Ma. Aubry et al. (1988) suggested that the Paleocene/Eocene boundary lies within Zone NP10 and assigned an age estimate of $57.0 \mathrm{Ma}$. At high latitudes, the last occurrence (LO) of the nannofossil Fasciculithus spp. and the first occurrence (FO) of the planktonic foraminifer Pseudohastigerina spp. are used to approximate the Paleocene/Eocene boundary. There are problems correlating the high-latitude Leg 114 sites precisely to the time scale because the LO of Fasciculithus spp. postdates the Paleocene/ Eocene boundary of Berggren et al. (1985) by 0.4 m.y., while the FO of Pseudohastigerina spp. is not well-calibrated to the time scale. Considering these biostratigraphic problems and the core recovery difficulties experienced on Leg 114, the Paleocene/Eocene boundary is difficult to locate precisely at the sites studied here.

Samples examined for benthic foraminiferal faunal and isotopic analyses were washed with sodium metaphosphate $(5.5 \mathrm{~g} / \mathrm{L})$ and/or hydrogen peroxide ( $3 \%$ solution) in tap water through a $63-\mu \mathrm{m}$ sieve and air dried. Benthic foraminifers were picked from aliquots of size fraction greater than $149 \mu \mathrm{m}$ 
and mounted on reference slides. In general, 100-400 specimens were picked per sample. In addition, some shipboard core-catcher samples were used for qualitative range chart information. The benthic foraminifers were identified using the taxonomy of Tjalsma and Lohmann (1983) and van Morkhoven et al. (1986). These studies form a comprehensive taxonomic base applicable to the Paleocene and Eocene of the Southern Ocean. We illustrate characteristic Paleocene (Pls. 1 and 2) and Eocene (Pls. 3 and 4) benthic foraminifers. We follow Berggren and Miller (in press) in recognizing the following faunal depth zones: upper bathyal $(200-600 \mathrm{~m})$, middle bathyal $(600-1000 \mathrm{~m})$, lower bathyal $(1000-2000 \mathrm{~m})$, upper abyssal (2000-3000 m), and lower abyssal (3000 m).

We compiled range charts and calculated extinction rates for the Paleocene to Eocene section from the benthic foraminiferal qualitative data for the Leg 114 sites. While most of our taxa are identified to the species level, we did not split up some of the genera, such as Lenticulina, Lagena, Fissurina, or Pleurostomella. These undifferentiated genera range throughout the Paleocene to Eocene section at our Leg 114 sites. Some genera may have species (usually rare and sporadic) that first occur or last occur within the section that we have not recorded. Therefore, our extinction rates are only approximations of the true faunal turnover.

We performed Q-mode principal component and Varimax factor analyses on the relative abundance (percentage) data using modifications of programs provided by Lohmann (1980). These programs utilize a cosine-theta matrix, standardizing each sample to unit length; they were modified to run on a Macintosh microcomputer.

For isotopic analyses, benthic foraminifers were ultrasonically cleaned for $5-10 \mathrm{~s}$ and roasted at $370^{\circ} \mathrm{C}$ in a vacuum. We analyzed samples of the benthic foraminiferal taxa Nuttallides truempyi and Cibicidoides spp. Carbon isotope comparisons are particularly sensitive to the benthic foraminiferal taxon chosen. Studies have shown that Cibicidoides accurately records deep-water $\delta^{13} \mathrm{C}$ variations (e.g., Graham et al., 1981), and we have successfully used this taxon to reconstruct Oligocene-Miocene carbon isotope fluctuations (Miller and Fairbanks, 1985). Shackleton et al. (1984) found that $N$. truempyi yielded the same $\delta^{13} \mathrm{C}$ values as Cibicidoides, while the $\delta^{18} \mathrm{O}$ values were constantly offset from Cibicidoides by about $0.15 \%$; we analyzed paired samples of $N$. truempyi and Cibicidoides spp. to confirm this (see "Results" section). Isotope measurements were made using a Carousel- 48 automatic carbonate preparation device attached to a Finnigan MAT 251 (Table 2). Replicate samples yielded mean $\delta^{18} \mathrm{O}$ differences of $0.161 \%$ and mean $\delta^{13} \mathrm{C}$ differences of $0.161 \%$, respectively (Table 2 ).

Paleodepth estimates were calculated assuming simple thermal subsidence and empirical age-subsidence curves ("backtracking") (Sclater et al., 1971; Berger and Winterer, 1974; among others) of the following form.

For crust younger than $80 \mathrm{Ma}$ :

$$
\begin{gathered}
P d=I d+k t^{1 / 2}-S, \text { and } \\
I d=P r-k(\text { basement age })^{1 / 2}+S .
\end{gathered}
$$

For crust older than $80 \mathrm{Ma}$ :

$$
\begin{gathered}
P d=I d-A+A \cdot e^{(-t / \text { tau })}-S, \text { and } \\
I d=\operatorname{Pr}+A-A \cdot e^{-(\text {basement age/tau })}+\mathrm{S} .
\end{gathered}
$$

(where $P r=$ present depth; $P d=$ paleodepth; $I d=$ initial depth; $t=$ (age of basement minus the age of level considered); $S=$ sediment correction of 0.66 (basement depth below seafloor minus depth below seafloor of level considered); and $\operatorname{tau}=$ decay constant of 62.5.)

The constants $A$ and $k$ have been empirically determined for the Atlantic as -3650 and 300 , respectively (Miller et al., 1987b). Basement depths, basement ages, initial depths, equations used are provided in the Figure 3 caption and the paleodepths are listed in Table 3.

We compared these backtracked paleodepths to paleodepth estimates based on benthic foraminiferal assemblage composition (Table 3). Tjalsma and Lohmann (1983) constructed age and depth distributions of deep-water benthic foraminifers from 48 Paleocene and 77 Eocene samples recovered from the Atlantic and Caribbean. This study estimates paleodepths for Sites $698,699,700$, and 702 based on Tjalsma and Lohmann's (1983) age-depth compilations for Paleocene and Eocene benthic foraminifers and compares these estimates with backtracked paleobathymetric values calculated assuming simple thermal subsidence (Table 3 ). The assumption here is that early Paleogene faunal distributions were not grossly different in the Southern Ocean compared to Tjalsma and Lohmann's (1983) Atlantic and Caribbean sites. As we show in the next section, some faunal patterns were different between these regions, and therefore this assumption is not entirely warranted. However, the faunal estimates and the backtracked depths show excellent agreement (Table 3); the exception is at Site 698 , where the backtracked paleodepths are shallower than those predicted by benthic foraminiferal assemblages. The close agreement implies that although some faunal distributions may have varied regionally, Southern Ocean early Paleogene bathymetric estimates based on benthic foraminiferal abundances are still reliable.

\section{RESULTS}

\section{Paleobathymetry}

The late Paleocene and early Eocene backtracked paleodepths at Hole 698A are about 800 and $900 \mathrm{~m}$, respectively (Fig. 3); this is the only location examined in this study that shows a discrepancy between the backtracked paleodepths and the faunal estimates. Faunal data suggest that Hole $698 \mathrm{~A}$ was situated in a lower bathyal setting (1500 $\pm 500 \mathrm{~m}$ ) in the Paleocene, based on moderate to high abundances of Stensioina beccariiformis, Lenticulina spp., Anomalinoides danicus, and Pullenia coryelli. Similarly, the Eocene assemblages indicate paleodepths of 1000-2000 m with moderate to high abundances of Cibicidoides praemundulus, Cibicidoides eocaenus, Anomalinoides capitatus, Hanzawaia ammophilus, Lenticulina spp., Osangularia mexicana, and buliminids, along with very low abundances of deep-water species Alabamina dissonata and Abyssamina poagi.

The backtracked depths at Hole 702B range from approximately $1800 \mathrm{~m}$ (early Eocene) to approximately $2250 \mathrm{~m}$ (late Eocene) (Fig. 3). The late Paleocene and Eocene benthic foraminiferal biofacies at Hole 702B indicate paleodepths of 1000-2000 m (lower bathyal). Paleocene biofacies contain moderate to high abundances of Cibicidoides hyphalus, Lenticulina spp., and buliminids; $S$. beccariiformis is more abundant than Nuttallides truempyi. The Eocene fauna contains high abundances of $C$. eocaenus, Lenticulina spp., and buliminids (in particular, Bulimina callahani and Bulimina semicostata) and very low abundances of primarily deeper water species such as Abyssamina spp., A. dissonata, and Clinapertina spp. 
Table 2. Benthic foraminiferal stable isotope values, Holes 698A, 699A, 700B, 702B and DSDP Site 98.

\begin{tabular}{|c|c|c|c|c|}
\hline $\begin{array}{l}\text { Core, section, } \\
\text { interval }(\mathrm{cm})\end{array}$ & $\begin{array}{l}\text { Depth } \\
\text { (mbsf) }\end{array}$ & $\begin{array}{l}\text { Age } \\
\text { (Ma) }\end{array}$ & $\delta^{18} \mathrm{O}_{\mathrm{PDB}}$ & $\delta^{13} \mathrm{C}_{\mathrm{PDI}}$ \\
\hline \multicolumn{5}{|l|}{ 114-698A- } \\
\hline \multicolumn{5}{|c|}{ Nuttallides truempyi } \\
\hline $6 \mathrm{R}-\mathrm{CC}$ & 45.20 & 55.54 & -0.285 & 0.524 \\
\hline 7R-CC & 51.50 & 56.08 & -0.306 & 0.321 \\
\hline $8 \mathrm{R}-\mathrm{CC}$ & 62.80 & 57.05 & -0.056 & 0.882 \\
\hline $9 \mathrm{R}-2,17-21$ & 72.17 & 58.06 & -0.053 & 0.856 \\
\hline 9R-CC & 73.40 & 58.21 & 0.297 & 2.195 \\
\hline \multicolumn{5}{|c|}{ Cibicidoides spp. } \\
\hline 2R-1, 86-90 & 4.86 & 52.07 & -0.476 & 1.141 \\
\hline 2R-2, 87-91 & 6.37 & 52.20 & -0.617 & 0.857 \\
\hline $2 \mathrm{R}-3,23-27$ & 7.23 & 52.28 & -0.674 & 0.737 \\
\hline $3 R-1,60-64$ & 14.10 & 52.87 & -0.631 & 0.906 \\
\hline $4 \mathrm{R}-1,82-86$ & 23.82 & 53.70 & -0.774 & 0.535 \\
\hline $5 R-1,68-72$ & 33.18 & 54.50 & -0.811 & 0.321 \\
\hline $5 R-2,68-72$ & 34.68 & 54.63 & -0.782 & 0.482 \\
\hline $6 \mathrm{R}-1,140-144$ & 43.40 & 55.38 & -0.499 & 0.489 \\
\hline $6 R-2,32-36$ & 43.82 & 55.42 & -0.560 & 0.261 \\
\hline $9 \mathrm{R}-1,61-65$ & 71.11 & 57.93 & -0.223 & 1.052 \\
\hline 10R-1, 144-148 & 81.44 & 59.22 & 0.066 & 1.873 \\
\hline $10-2,21-25$ & 81.71 & 59.26 & 0.390 & 2.433 \\
\hline
\end{tabular}

114-699A-

\begin{tabular}{lllll}
\multicolumn{5}{c}{ Nuttallides truempyi } \\
48X-3, 34-38 & 442.94 & 52.46 & -0.210 & 0.226 \\
$48 X-3,69-73$ & 443.28 & 52.49 & -0.938 & 1.029 \\
$49 X-3,38-42$ & 452.45 & 53.30 & -0.507 & 0.672 \\
& & & -0.970 & 0.738 \\
50X-3, 104-108 & 462.64 & 54.19 & -0.699 & 1.184 \\
51X-CC & 468.10 & 54.67 & -1.246 & 0.625 \\
52X-CC & 477.60 & 55.51 & -1.423 & 0.302 \\
53X-CC & 487.10 & 56.34 & -1.490 & 0.279 \\
54X-2, 22-26 & 498.32 & 57.33 & -1.323 & 1.487
\end{tabular}

$114-700 \mathrm{~B}-$

\begin{tabular}{lccrr}
\multicolumn{5}{c}{ Nuttallides truempyi } \\
14R-CC & 131.20 & 50.60 & -0.462 & 0.925 \\
26R-1, 60-64 & 238.60 & 57.85 & -0.652 & 2.072 \\
26R-2, 60-64 & 240.10 & 57.98 & -0.847 & 2.215 \\
26R-CC & 241.95 & 58.14 & -0.941 & 2.149 \\
27R-1, 86-90 & 248.36 & 58.68 & -0.921 & 2.136 \\
27R-CC & 249.30 & 58.76 & -0.833 & 2.532 \\
28R-1, 48-52 & 257.48 & 59.46 & -0.699 & 2.788 \\
28R-4, 48-52 & 261.98 & 59.84 & -0.154 & 2.068 \\
29R-CC & 269.35 & 60.47 & -0.550 & 1.890 \\
30R-5, 80-84 & 282.80 & 61.61 & -0.837 & 2.027 \\
31R-4, 49-53 & 290.49 & 62.27 & -0.862 & 1.711 \\
32R-4, 55-59 & 300.05 & 63.08 & -0.925 & 1.698 \\
34R-3,82-86 & 317.82 & 64.59 & -1.366 & 1.878 \\
36R-3, 18-22 & 329.18 & 65.56 & -1.358 & 2.045 \\
& & & & \\
7R-2, 127-131 & 67.17 & 46.54 & 0.209 & 1.008 \\
7R-5, 127-131 & 71.67 & 46.85 & 0.259 & 0.983 \\
8R-1, 139-143 & 75.29 & 47.10 & 0.369 & 0.841 \\
8R-3, 139-143 & 78.29 & 47.30 & 0.346 & 0.729 \\
9R-2, 68-72 & 85.58 & 47.80 & 0.436 & 0.897 \\
9R-4, 68-72 & 88.58 & 48.00 & 0.446 & 0.675 \\
10R-1,60-64 & 93.50 & 48.34 & 0.270 & 0.794 \\
10R-3,60-64 & 96.50 & 48.54 & 0.239 & 0.919 \\
11R-1, 17-21 & 102.57 & 48.95 & 0.148 & 0.624 \\
13R-2, 88-92 & 123.78 & 50.40 & -0.497 & 0.612 \\
13R-5, 89-93 & 128.29 & 50.70 & -0.444 & 0.778 \\
14R-CC & 131.20 & 50.90 & -0.330 & 1.154 \\
16R-2, 110-114 & 152.50 & 52.35 & -0.491 & 1.418 \\
16R-6, 110-114 & 158.50 & 52.76 & -0.912 & 0.807 \\
18R-1, 129-133 & 170.19 & 53.56 & -0.584 & 1.061 \\
18R-4, 119-123 & 174.59 & 53.85 & -0.957 & 0.436 \\
20R-1, 72-76 & 188.62 & 54.77 & -0.849 & 0.443 \\
20R-3,64-68 & 191.54 & 54.96 & -0.596 & 0.667 \\
20R-CC & 191.70 & 54.97 & -0.767 & 0.504 \\
21R-3, 70-74 & 196.60 & 55.29 & -0.924 & -0.001 \\
21R-4, 19-23 & 197.59 & 55.36 & -0.771 & 0.437
\end{tabular}

Table 2 (continued).

\begin{tabular}{lcccc}
\hline $\begin{array}{c}\text { Core, section, } \\
\text { interval }(\mathrm{cm})\end{array}$ & $\begin{array}{c}\text { Depth } \\
\text { (mbsf) }\end{array}$ & $\begin{array}{c}\text { Age } \\
(\mathrm{Ma})\end{array}$ & $\delta^{18} \mathrm{O}_{\text {PDB }}$ & $\delta^{13} \mathrm{C}_{\text {PDB }}$ \\
\hline 21R-CC & 197.90 & 55.38 & -0.631 & 0.416 \\
26R-2, 60-64 & 240.10 & 58.61 & -0.655 & 2.147 \\
26R-CC & 242.00 & 58.81 & -0.578 & 2.392 \\
27R-1, 86-90 & 248.36 & 59.36 & -0.827 & 1.902 \\
27R-CC & 249.30 & 59.41 & -0.501 & 2.901 \\
28R-1, 48-52 & 257.48 & 59.89 & -0.493 & 2.854 \\
29R-2, 98-102 & 268.98 & 60.56 & -0.137 & 2.060 \\
29R-CC & 269.49 & 60.58 & -0.210 & 1.921 \\
30R-5, 80-84 & 282.80 & 61.36 & -0.783 & 2.128 \\
31R-4, 49-53 & 290.49 & 61.80 & -0.562 & 1.789
\end{tabular}

114-702B-

\begin{tabular}{lrrrr}
\multicolumn{5}{c}{ Nuttallides truempyi } \\
a 4 X-7, 68-72 & 32.23 & 39.50 & 0.882 & 0.751 \\
$9 \mathrm{X}-1,110-114$ & 73.90 & 42.87 & -0.035 & 0.826 \\
$9 \mathrm{X}-5,110-114$ & 79.90 & 43.29 & 0.208 & 0.108 \\
$10 \mathrm{X}-3,40-44$ & 85.70 & 43.90 & 0.271 & 0.558 \\
$11 \mathrm{X}-3,80-84$ & 95.60 & 44.51 & 0.064 & 0.696 \\
$12 \mathrm{X}-1,58-62$ & 101.88 & 44.99 & 0.110 & 0.503 \\
$13 \mathrm{X}-1,60-64$ & 111.40 & 45.96 & 0.164 & 0.905 \\
$13 \mathrm{X}-3,60-64$ & 114.40 & 46.23 & 0.237 & 0.791 \\
$15 \mathrm{X}-1,70-74$ & 130.50 & 47.17 & 0.353 & 0.662 \\
$15 \mathrm{X}-4,70-74$ & 135.00 & 47.44 & 0.198 & 0.539 \\
$16 \mathrm{X}-6,60-64$ & 147.40 & 48.17 & 0.015 & 0.472 \\
$17 \mathrm{X}-2,120-124$ & 151.50 & 48.41 & 0.151 & 0.786 \\
$17 \mathrm{X}-5,120-124$ & 156.00 & 48.67 & 0.081 & 0.554 \\
$22 \mathrm{X}-1,62-66$ & 196.92 & 53.17 & -0.782 & 0.934 \\
$22 \mathrm{X}-2,62-66$ & 198.42 & 53.41 & -0.828 & 0.777 \\
$26 \mathrm{X}-\mathrm{CC}$ & 235.40 & 57.00 & -0.069 & 1.042 \\
$27 \mathrm{X}-1,12-16$ & 243.92 & 58.19 & -0.490 & 0.631 \\
$27 \mathrm{X}-\mathrm{CC}$ & 245.10 & 58.41 & -0.051 & 1.328 \\
$29 \mathrm{X}-\mathrm{CC}$ & 263.30 & 59.56 & 0.149 & 2.155 \\
$30 \mathrm{X}-2,30-34$ & 274.10 & 59.84 & -0.053 & 2.262 \\
& & & 0.013 & 2.230
\end{tabular}

$\begin{array}{lrrrr}\text { a 3H-6, 68-72 } & 21.23 & 38.62 & 0.842 & 0.978 \\ \text { a } 4 \text { X-2, 68-72 } & 24.73 & 38.90 & 0.893 & 0.934 \\ & & & 0.857 & 0.939 \\ \text { a } 4 \text { X-4, 68-72 } & 27.73 & 39.14 & 0.781 & 0.868 \\ \text { a 4X-7, 68-72 } & 32.23 & 39.50 & 1.010 & 1.076 \\ 5 X-2,80-84 & 37.10 & 39.89 & 0.722 & 1.057 \\ 5 X-4,64-68 & 39.94 & 40.11 & 0.994 & 0.853 \\ 6 \text { X-2, 100-104 } & 46.80 & 40.66 & 0.904 & 0.860 \\ 8 X-3,50-54 & 66.80 & 42.31 & 0.233 & 1.434 \\ 8 X-5,50-54 & 69.80 & 42.56 & 0.181 & 1.439 \\ 9 X-5,110-114 & 79.90 & 43.29 & 0.327 & 0.593 \\ 10 X-3,40-44 & 85.70 & 43.90 & 0.496 & 0.968 \\ 11 X-1,80-84 & 92.60 & 44.36 & 0.244 & 0.899 \\ 12 X-1,58-62 & 101.88 & 44.99 & 0.360 & 0.910 \\ 12 X-3,58-62 & 104.88 & 45.29 & 0.288 & 1.177 \\ 13 X-3,60-64 & 114.40 & 46.23 & 0.311 & 1.581 \\ 14 X-1,68-72 & 120.98 & 46.62 & 0.132 & 0.923 \\ 14 X-3,68-72 & 123.98 & 46.79 & 0.351 & 1.018 \\ 15 X-1,70-74 & 130.50 & 47.17 & 0.528 & 1.000 \\ 15 X-4,70-74 & 135.00 & 47.44 & 0.323 & 0.765 \\ 16 X-1,60-64 & 139.90 & 47.73 & 0.189 & 0.763 \\ 16 X-2,60-64 & 141.40 & 47.82 & 0.286 & 0.829 \\ 16 X-3,60-64 & 142.90 & 47.90 & 0.275 & 0.797 \\ 16 X-6,60-64 & 147.40 & 48.17 & 0.168 & 0.792 \\ 17 X-2,120-124 & 151.50 & 48.41 & 0.329 & 1.015 \\ 17 X-5,120-124 & 156.00 & 48.67 & 0.276 & 0.878 \\ 18 X-3,20-24 & 161.50 & 49.04 & 0.251 & 0.653 \\ 18 X-5,20-24 & 164.50 & 49.25 & 0.071 & 0.667 \\ 19 X-1,20-24 & 168.00 & 49.49 & -0.053 & 0.584 \\ 19 X-3,60-64 & 171.00 & 49.70 & -0.121 & 0.801 \\ 19 X-4,20-24 & 172.50 & 49.80 & -0.101 & 0.850 \\ 19 X-5,20-24 & 174.00 & 49.90 & -0.105 & 0.854 \\ 19 X-6,20-24 & 175.50 & 50.01 & -0.268 & 0.725 \\ 21 X-1,65-69 & 187.45 & 52.29 & -0.340 & 1.079 \\ 21 X-2,65-69 & 188.95 & 52.37 & -0.460 & 1.005 \\ 21 X-3,65-69 & 190.45 & 52.45 & -0.582 & 0.913 \\ 22 X-3,62-66 & 199.92 & 53.65 & -0.802 & 0.888 \\ 22 X-4,62-66 & 201.42 & 53.81 & -0.811 & 1.018 \\ 23 X-1,49-53 & 206.29 & 54.27 & -0.589 & 0.567 \\ 24 X-2,20-24 & 217.00 & 55.27 & -0.460 & 0.385\end{array}$


Table 2 (continued).

\begin{tabular}{lllrl}
\hline $\begin{array}{c}\text { Core, section, } \\
\text { interval }(\mathrm{cm})\end{array}$ & $\begin{array}{c}\text { Depth } \\
(\mathrm{mbsf})\end{array}$ & $\begin{array}{c}\text { Age } \\
(\mathrm{Ma})\end{array}$ & $\delta^{18} \mathrm{O}_{\text {PDB }}$ & $\delta^{13} \mathrm{C}_{\mathrm{PDB}}$ \\
\hline 24X-3, 20-24 & 218.50 & 55.42 & -0.379 & 0.468 \\
25X-1, 40-44 & 225.20 & 56.04 & -0.340 & 0.628 \\
26X-1, 59-63 & 234.89 & 56.95 & -0.285 & 0.641 \\
26X-CC & 235.40 & 57.00 & 0.018 & 1.327 \\
27X-CC & 245.10 & 58.41 & -0.017 & 1.663 \\
28X-CC & 253.70 & 59.31 & 0.106 & 2.434 \\
29X-CC & 263.30 & 59.56 & 0.105 & 2.574 \\
$30 X-1,30-34$ & 272.60 & 59.80 & -0.066 & 2.293 \\
& & & 0.026 & 2.864 \\
30X-2, 30-34 & 274.10 & 59.84 & -0.061 & 2.821 \\
& & & 0.106 & 2.809 \\
30X-CC & 274.80 & 59.85 & 0.243 & 2.601 \\
31X-1, 34-38 & 277.64 & 59.93 & 0.011 & 2.242 \\
31X-CC & 278.50 & 59.95 & -0.249 & 1.834 \\
32X-1, 36-40 & 287.16 & 60.17 & -0.065 & 1.858 \\
32X-CC & 287.60 & 60.18 & -0.071 & 1.806 \\
& & & 0.004 & 1.655
\end{tabular}

98-

\begin{tabular}{lllrl}
$6-3,63-65$ & 133.63 & 48.92 & 0.684 & 0.835 \\
$7-2,55-57$ & 169.05 & 52.19 & -0.071 & 0.672 \\
$7-3,146-148$ & 171.46 & 52.41 & -0.148 & 0.806 \\
$7-4,143-145$ & 172.93 & 52.55 & 0.035 & 0.904 \\
$7-5,132-134$ & 174.32 & 52.68 & -0.094 & 0.701 \\
$7-6,107-109$ & 175.57 & 52.79 & -0.252 & 0.563 \\
$11-2,121-123$ & 233.71 & 57.57 & -0.492 & 0.310 \\
$12-1,57-59$ & 240.57 & 58.03 & -0.427 & 0.424 \\
\hline
\end{tabular}

a Analyses from Hole 702A are corrected to sub-bottom depths for Hole $702 \mathrm{~B}$.

At Site 700B, backtracking indicates Paleocene depths of $2250 \mathrm{~m}$ and Eocene depths of $2500-3000 \mathrm{~m}$ (Fig. 3). Benthic biofacies suggest Paleocene depths of $2000-2500 \mathrm{~m}$ and Eocene depths of $2500-3000 \mathrm{~m}$. Paleocene biofacies contain comparable abundances of $S$. beccariiformis and $N$. truempyi, along with moderate abundances of Aragonia spp., Lenticulina spp., Tritaxia spp., and buliminids. Early Eocene biofacies contain moderate to high abundances of buliminids, Clinapertina spp., A. dissonata, and A. poagi and low abundances of Lenticulina spp. The middle Eocene disappearance of a buliminid assemblage at Hole $700 \mathrm{~B}$ suggests that this location may have subsided below about $2750-3000 \mathrm{~m}$ by this time, slightly deeper than the backtracked estimate of $2600-2700 \mathrm{~m}$ (Fig. 3). However, the apparently coeval disappearance of the buliminid assemblage at the shallower Hole 702B (see previous paragraph) suggests that this may be the result of oceanographic changes rather than subsidence.

At Hole 699A, backtracked paleodepths are approximately $2700 \mathrm{~m}$ (Paleocene) and $2900 \mathrm{~m}$ (Eocene) (Fig. 3). These estimates show good agreement with benthic foraminiferal biofacies depth estimates of 2000-3000 m (upper abyssal zone) for the Paleocene and approximately $2500 \mathrm{~m}$ for the
Eocene. Paleocene faunal depth estimates are indicated by the dominance of $N$. truempyi over $S$. beccariiformis, high abundances of $C$. hyphalus, and low abundances of Lenticulina spp. Eocene estimates are based on moderate abundances of A. dissonata and low abundances of Lenticulina spp., Abyssamina spp., and Clinapertina spp.

\section{Faunal Changes}

The benthic foraminiferal assemblages examined from the four Leg 114 sites document that the late Paleocene/early Eocene extinction event was recorded in the Atlantic sector of the Southern Ocean over a wide bathymetric range. Fifteen species last occur in the upper Paleocene in at least three of the four sites examined (Table 4): Alabamina creta, Anomalinoides danicus, Anomalinoides praeacuta, Aragonia velascoensis, Bolivinoides delicatulus, Bulimina velascoensis, Buliminella beaumonti, Cibicidoides hyphalus, Dorothia trochoides, Gyroidinoides quadratus, Neoeponides hillebrandti, Neoflabellina semireticulata, Pullenia coryelli, Spiroplectammina jarvisi, and Stensioina beccariiformis. These are the same taxa that disappeared from coeval levels in the Atlantic and Pacific oceans (Tjalsma and Lohmann, 1983; Miller et al., 1987c).

\section{Site 698}

It is difficult to locate precisely the Paleocene/Eocene boundary at Site 698 (850 m backtracked paleodepth; Fig. 3). However, it is clear that 17 taxa last appear between the LO of Fasciculithus spp. (71.02-62.84 m below seafloor [mbsf]) (Shipboard Scientific Party, 1988a) and the FO of Pseudohastigerina spp. (62.64-61.15 mbsf) (P. F. Ciesielski, unpubl. data) (Fig. 4 and Table 4). Only 11 Paleocene taxa survive across the Paleocene/Eocene boundary, representing a possible extinction rate of $59 \%$. S. beccariiformis is present consistently up to the level of the LO of Fasciculithus spp. Several isolated specimens occur between the LO of Fasciculithus spp. and the FO of Pseudohastigerina spp. (Fig. 4). Other typical Paleocene taxa disappear near this interval (Table 4). Benthic foraminiferal species were not quick to rediversify after the extinction event. Taxa appeared gradually from the latest Paleocene through the early Eocene at Hole 698A (Table 4).

Factor analysis of the benthic foraminiferal relative abundance data from Hole 698A shows that $S$. beccariiformis dominates the Paleocene assemblages (Fig. 5). This fauna is replaced by an assemblage characterized by Cibicidoides $\mathrm{cf}$. pseudoperlucidus, $N$. truempyi, and Oridorsalis spp. in the lowermost Eocene. Turrilina robertsi becomes dominant in the lower Eocene, and in turn is replaced by Cibicidoides praemundulus near the lower/middle Eocene boundary (Fig. 5).

\section{Site 702}

At Site 702 (about 1700 to $2250 \mathrm{~m}$ paleodepth; Fig. 3), 17 benthic foraminiferal taxa disappear just below the LO of Fasciculithus spp. (243.86-235.77 mbsf) (P. F. Ciesielski,

Table 3. Comparison of backtracked paleodepth values and benthic foraminiferal faunal depth estimates for Leg 114 Paleogene locations.

\begin{tabular}{|c|c|c|c|c|c|c|}
\hline \multirow[b]{2}{*}{ Site } & \multicolumn{2}{|c|}{ Paleocene } & \multicolumn{2}{|c|}{ early Eocene } & \multicolumn{2}{|c|}{ middle Eocene } \\
\hline & Faunal & Backtracked & Faunal & Backtracked & Faunal & Backtracked \\
\hline 698 & $1500 \pm 500$ & 800 & $1500 \pm 500$ & 900 & & \\
\hline 699 & $2000-3000$ & 2700 & 2500 & 2900 & & \\
\hline 700 & $2000-2500$ & 2250 & $2500-3000$ & $2400-2600$ & $2750-3000$ & $2600-2700$ \\
\hline 702 & $1000-2000$ & $1700-1800$ & $1000-2000$ & $1800-2000$ & $1000-2000$ & $2000-2200$ \\
\hline
\end{tabular}

NOTE: Backtracked depths were computed using text equations (1)-(4) and parameters given in Figure 3. 
Table 4. Upper Paleocene to Eocene first and last occurrences of taxa, Holes 698A, 699A, $700 \mathrm{~B}$, and $702 \mathrm{~B}$.

\begin{tabular}{ll}
\hline \multicolumn{1}{c}{ Occurrence } & Taxa \\
\hline Hole 698A & Alabamina creta, Anomalinoides danicus, Anomalinoides \\
Last occurrence in the & praeacuta, Aragonia velascoensis, Bulimina trihedra, \\
Paleocene & Bulimina velascoensis, Buliminella beaumonti, Cibicidoides \\
& hyphalus, Dorothia trochoides, Gyroidinoides globulosus, \\
& Neoflabellina semireticulata, Pullenia coryelli, Pyramidina \\
rutida, Spiroplectammina jarvisi, Stensioina beccariiformis, \\
Tappanina selmensis \\
Alabamina dissonata, Anomalinoides capitatus, \\
Anomalinoides semicribratus, Anomalinoides spissiformis, \\
uppermost Paleocene to \\
Aragonia aragonensis, Bulimina semicostata, Buliminella \\
grata, Cibicidoides praemundulus, Cibicidoides eocaenus, \\
Cibicidoides grimsdalei, Cibicidoides aff. subspiratus, \\
Clinapertina spp., Globocassidulina subglobosa, Hanzawaia \\
ammophilus
\end{tabular}

Hole 699A

Last occurrence in the upper

Paleocene

First occurrence in the lower Eocene

Hole 700B

Last occurrence in the upper Paleocene

First occurrence in the lower to middle Eocene

Hole 702B

Last occurrence in the Paleocene

First occurrence in the uppermost Paleocene

First occurrence in the lower Eocene

First occurrence in the lower middle Eocene

First occurrence in the upper middle Eocene
Alabamina creta, Anomalinoides praeacuta, Bolivinoides delicatulus, Buliminella beaumonti, Bulimina midwayensis, Cibicidoides hyphalus, Cibicidoides $\mathrm{cf}$. pseudoperlucidus, Dorothia trochoides, Gyroidinoides globulosus, Gyroidinoides quadratus, Neoeponides hillebrandti, Pullenia coryelli, Spiroplectammina jarvisi, Stensioina beccariiformis, Tritaxia spp.

Abysamina spp., Alabamina dissonata, Anomalinoides capitatus, Anomalinoides spissiformis, Aragonia aragonensis, Bulimina semicostata, Bulimina thanetensis, Bulimina trinitatensis, Buliminella grata, Cibicidoides eocaenus, Cibicidoides havanensis, Cibicidoides praemundulus, Clinapertina spp., Globocassidulina subglobosa, Hanzawaia ammophilus, Stilostomella spp.

Alabamina creta, Anomalinoides danicus, Anomalinoides praeacuta, Aragonia velascoensis, Bolivinoides delicatulus, Bulimina midwayensis, Bulimina velascoensis, Buliminella beaumonti, Cibicidoides hyphalus, Cibicidoides velascoensis, Dorothia trochoides, Gyroidinoides quadratus, Neoeponides lunata, Neoeponides hillebrandti, Neoflabellina semireticulata, Pullenia coryelli, Spiroplectammina jarvisi, Stensioina beccarifformis, Tritaxia paleocenica, Tritaxia havanensis

Alabamina dissonata, Anomalinoides semicribratus, Aragonio aragonensis, Bulimina alazanensis, Bulimina semicostata, Buliminella grata, Cibicidoides bradyi, Cibicidoides havanensis, Clinapertina spp., Globocassidulina subglobosa, Hanzawaia ammophilus, Pullenia bulloides, Nuttallides umbonifera, Turrilina robertsi, Vulvulina spinosa

Alabamina creta, Anomalinoides danicus, Aragonia velascoensis, Bolivinoides delicatulus, Bulimina midwayensis, Bulimina velascoensis, Buliminella beaumonti, Cibicidoides hyphalus, Cibicidoides velascoensis, Dorothia trochoides, Gyroidinoides quadratus, Neoeponides hillebrandtillunata, Neoflabellina semireticulata, Pullenia coryelli, Stensioina beccarïformis, Tritaxia havanensis, Tritaxia paleocenica (Anomalinoides praeacuta disappears in the lowermost Eocene)

Aragonia aragonensis, Cibicidoides eocaenus, Cibicidoides praemundulus, Tappanina selmensis, Turrilina robertsi

Alabamina dissonata, Bulimina callahani, Bulimina semicostata, Buliminella grata, Clinapertina spp., Globocassidulina subglobosa, Hanzawaia ammophilus, Karreriella subglabra, Pullenia bulloides, Spiroplectammina spectabilis

Anomalinoides semicribratus, Bulimina alazanensis, Cibicidoides grimsdalei, Karreriella chapapotensis

Bulimina macilenta, Cibicidoides bradyi, Nuttallides umbonifera, Turrilina brevispira 


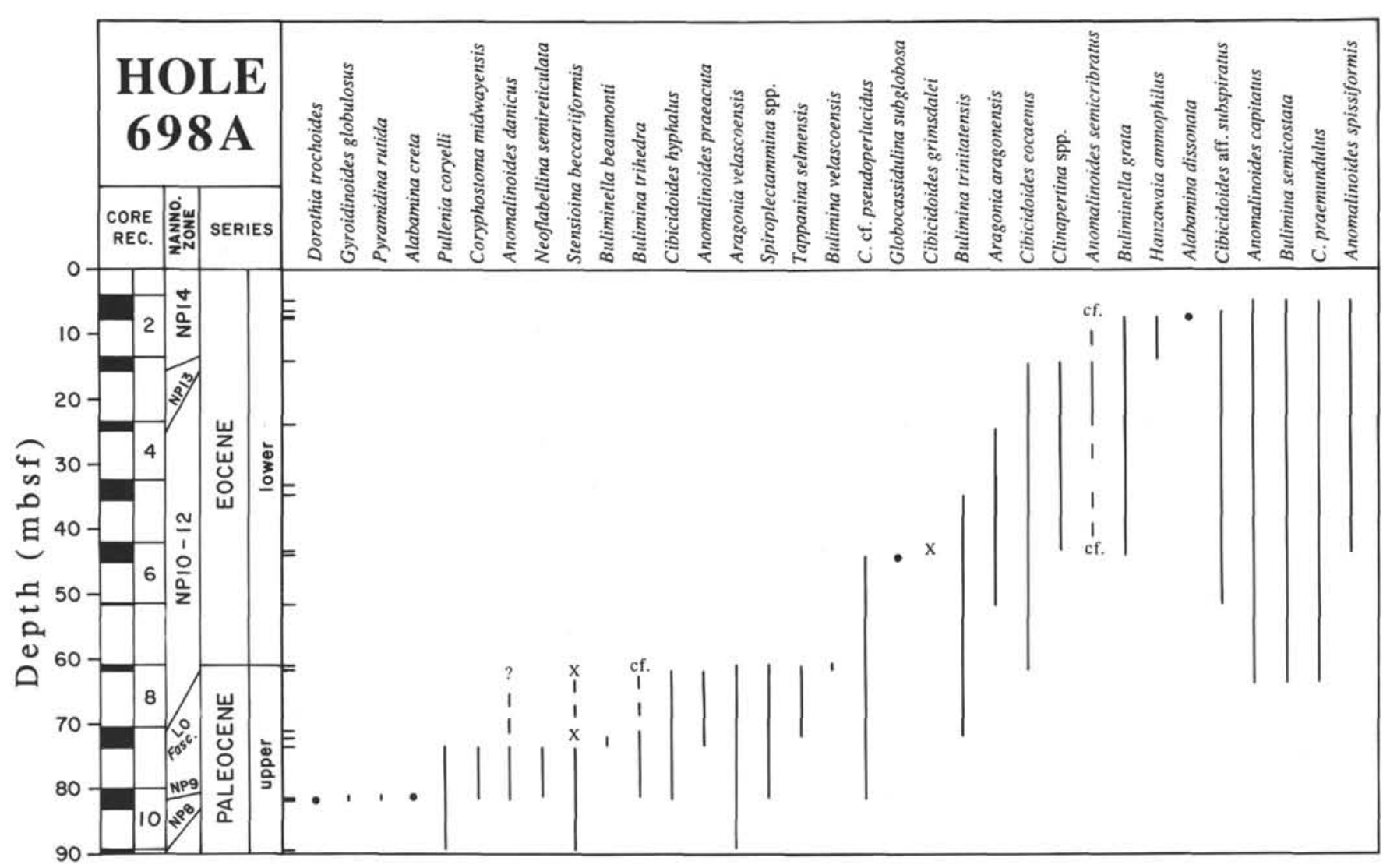

Figure 4. Ranges of benthic foraminifers at Hole 698A. $\times=$ single, isolated specimen; solid circles $=$ more than one specimen of a taxon that is present in only one sample; dashed line = uncertain or possible range. Tick marks indicate samples examined for benthic foraminiferal stratigraphic ranges. LO Fasc. = last occurrence of the nannofossil Fasciculithus spp. Nannofossil biostratigraphy after Shipboard Scientific Party (1988a).

unpubl. data). Seventeen taxa survive the faunal turnover at Hole 702B, yielding an extinction rate of $50 \%$ across the Paleocene/Eocene boundary (Fig. 6 and Table 4). The radiation of benthic foraminiferal species at Hole 702B occurs across nearly $90 \mathrm{~m}$ of section from the uppermost Paleocene through the lower middle Eocene. Five taxa first appear in the uppermost Paleocene, while 10 taxa first appear in the lower Eocene (Table 4). In contrast, we note that only four additional taxa first appear in the lower middle Eocene, and four additional taxa first appear in the upper middle Eocene (Fig. 6 and Table 4).

Factor analysis of the benthic foraminiferal relative abundance data from Hole 702B shows the dramatic decline of the Paleocene fauna characterized by $S$. beccariiformis, Gyroidinoides spp. (including the Paleocene forms G. globulosus and $G$. quadratus), and $C$. cf. pseudoperlucidus and its replacement by a $N$. truempyi-dominated assemblage in the Eocene (Fig. 7). This Eocene assemblage is replaced in a thin interval in the lower middle Eocene by a Bulimina semicostata, Lenticulina spp., and Oridorsalis spp. fauna. In the uppermost middle Eocene, the $N$. truempyi-dominated assemblage is replaced by an Oridorsalis spp., C. praemundulus, and Nuttallides umbonifera fauna; this change has been noted throughout the Atlantic (Tjalsma and Lohmann, 1983; Miller et al., 1985; Wood et al., 1985) and in the Pacific (Corliss and Keigwin, 1986).

\section{Site 700}

At Site 700 (2275 m paleodepth; Fig. 3), a preponderance of Paleocene benthic foraminiferal taxa disappear at the LO of Fasciculithus spp., although poor core recovery leaves a gap in the faunal record across the Paleocene/Eocene boundary (Fig. 8). Twenty-one taxa last appear in the uppermost $\mathrm{Pa}$ leocene, whereas 16 taxa survive the boundary extinction event (Fig. 8 and Table 4). This represents an extinction rate of $57 \%$. The radiation of benthic foraminifers after the $\mathrm{Pa}-$ leocene/Eocene extinctions was also gradual at Hole $700 \mathrm{~B}$, where 16 benthic foraminiferal taxa first appear across $160 \mathrm{~m}$ spanning the lower to middle Eocene (Table 4).

Factor analysis of the benthic foraminiferal relative abundance data from Hole $700 \mathrm{~B}$ shows that $S$. beccariiformis dominates the Paleocene assemblages (Fig. 9). A fauna characterized by Clinapertina spp., Alabamina dissonata, Buliminella grata, and Cibicidoides eocaenus dominates lowermost Eocene sediments. This is replaced in the lower Eocene by an assemblage characterized by $N$. truempyi, Oridorsalis spp., $C$. praemundulus, and B. semicostata.

\section{Site 699}

There was only one Paleocene sample available from Hole 699A (about 2700-2900 m paleodepth; Fig. 3), in which there is good agreement between the LO of Fasciculithus spp. and 
FACTOR 3

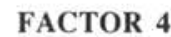

FACTOR 2

FACTOR 1

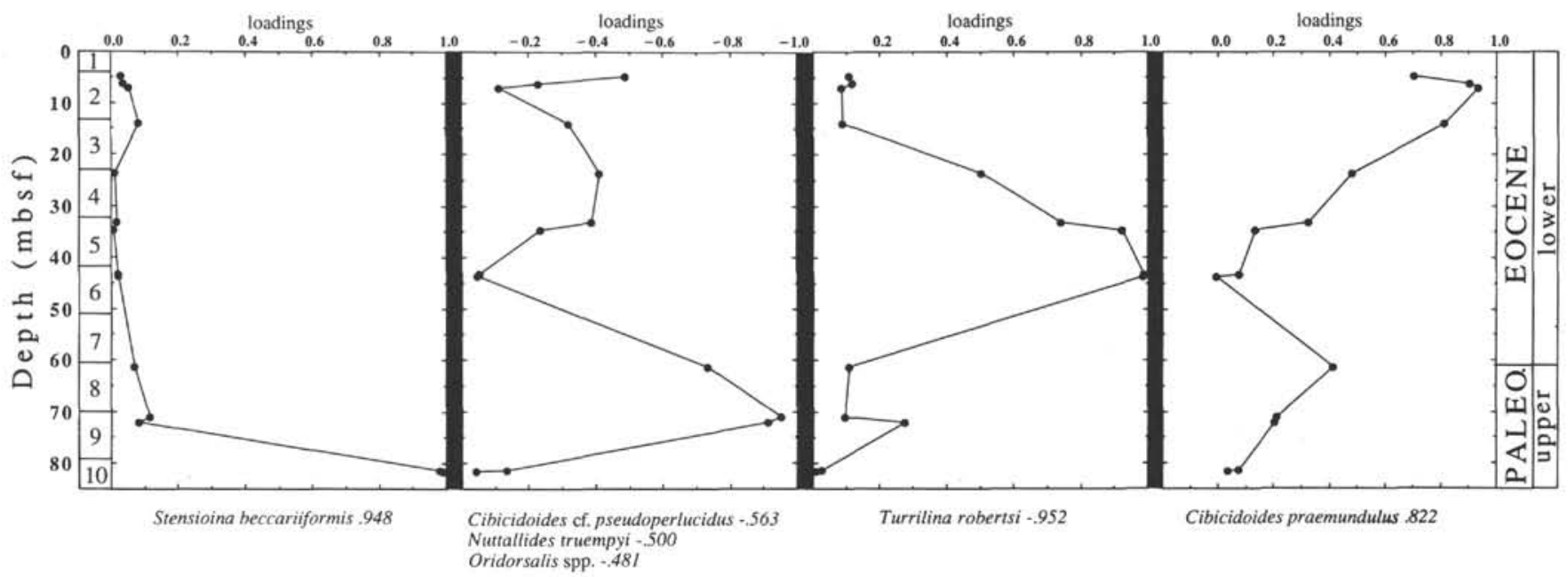

Figure 5. Q-mode Varimax factor analysis of benthic foraminiferal relative abundance data from Hole 698A. Factors are arranged in stratigraphic sequence of successive assemblages. Core numbers are to the left. Species contributing to each factor and their factor scores are indicated under each column. Factor 1 explains $25 \%$ of the variance, factor 2 explains $27 \%$ of the variance, factor 3 explains $14 \%$ of the variance, and factor 4 explains $22 \%$ of the variance.

the last appearances of Paleocene benthic foraminiferal taxa. Fifteen benthic foraminiferal taxa appear only in the $\mathrm{Pa}$ leocene sample at Hole 699A, while 12 taxa survive into the Eocene (Fig. 10 and Table 4). Assuming that the single Paleocene sample is representative, the possible extinction rate at Hole $699 \mathrm{~A}$ is $56 \%$. Sixteen taxa first appear in the $20 \mathrm{~m}$ of lower Eocene at Hole 699A (Table 4).

The second Q-mode principal component shows that the principal axis of faunal variation delineates the Paleocene sample dominated by $C$. cf. pseudoperlucidus, $C$. hyphalus, and $S$. beccariiformis from a typical Eocene assemblage (Fig. 11) at Hole 699A.

\section{Leg 114 Biofacies}

A Q-mode principal components analysis was performed on the combined relative abundances from Holes 698A, 699A, $700 \mathrm{~B}$, and $702 \mathrm{~B}$. The second principal component loadings were contoured on an age-paleodepth figure (Fig. 12). The second principal component delineates the major faunal dichotomy between a Paleocene Stensioina beccariiformisdominated assemblage and an Eocene assemblage. The late Paleocene fauna was fairly uniform across the depth range covered by the four sites examined in this study. The Eocene assemblages displayed greater depth-related diversity and are mapped based on the factor analyses of the Eocene data sets from the individual sites (Fig. 12). The early to middle Eocene at all Leg 114 locations was dominated by Nuttallides truempyi. In addition, several depth-distinctive taxa were important:

1. At the shallowest location (Hole 698A), Turrilina robertsi dominated the early part of the Eocene and was replaced by Cibicidoides praemundulus in the later early Eocene.

2. Bulimina semicostata became important in the late early to early middle Eocene at Holes $700 \mathrm{~B}$ and $702 \mathrm{~B}$.

3. Clinapertina and Alabamina dissonata joined N. truempyi in dominating the deepest locations in the early Eocene (Holes 700B and 699A).

4. N. truempyi declined in abundance during the late middle Eocene at Holes $700 \mathrm{~B}$ and $702 \mathrm{~B}$ (Fig. 12); Oridorsalis spp., C. praemundulus, and Nuttallides umbonifera replaced the $N$. truempyi-dominated assemblage at these sites.

Tjalsma and Lohmann (1983) constructed a similar biofacies summary. In their Atlantic data, the $S$. beccariiformisdominated assemblage was restricted to progressively shallower depths during the late Paleocene as it was replaced by the deeper $N$. truempyi fauna. The Leg 114 data do not show the same trend; rather, the $S$. beccariiformis-dominated assemblage survived until near the end of the Paleocene at all depths in the Atlantic sector of the Southern Ocean (Fig. 12), although it may have disappeared from the shallower sites slightly earlier than from the deeper sites. Not only did $S$. beccariiformis linger on in even the deepest locations of the Southern Ocean longer than it did in the Atlantic, but it also occurred in the highest abundances at Southern Ocean locations over a wide bathymetric range. A comparison of Atlantic, Pacific, and Southern Ocean relative abundances of $S$. beccariiformis shows that it is present in percentages greater than $20 \%$ up to nearly $60 \%$ only in the Southern Ocean (Fig. 13). This taxon probably preferred the Southern Ocean, although this cannot be determined from the relative abundance data because of the closed sum problem (e.g., Miller and Katz, 1987). The wide bathymetric extent of the high abundances of $S$. beccariiformis in our depth transect suggests that the late Paleocene Southern Ocean was vertically well mixed; stable isotope data also support this suggestion that the Southern Ocean was well mixed (this study).

\section{Isotope Stratigraphy and Paleoceanography}

Relatively complete upper Paleocene to Eocene sections recovered from Holes $700 \mathrm{~B}$ and $702 \mathrm{~B}$ provide the opportunity to monitor early Paleogene deep-water isotopic changes in the Atlantic sector of the Southern Ocean. The most complete record is afforded by Hole 702B, where the upper Paleocene to upper Eocene is nearly $300 \mathrm{~m}$ thick. Despite the poor recovery of the upper Paleocene, the Paleocene to Eocene section yielded well-preserved specimens of Cibicidoides spp. and Nuttallides truempyi suitable for oxygen and carbon stable isotope analysis. The Paleocene to Eocene section at Hole $700 \mathrm{~B}$ also suffers from poor recovery; in addition, the 


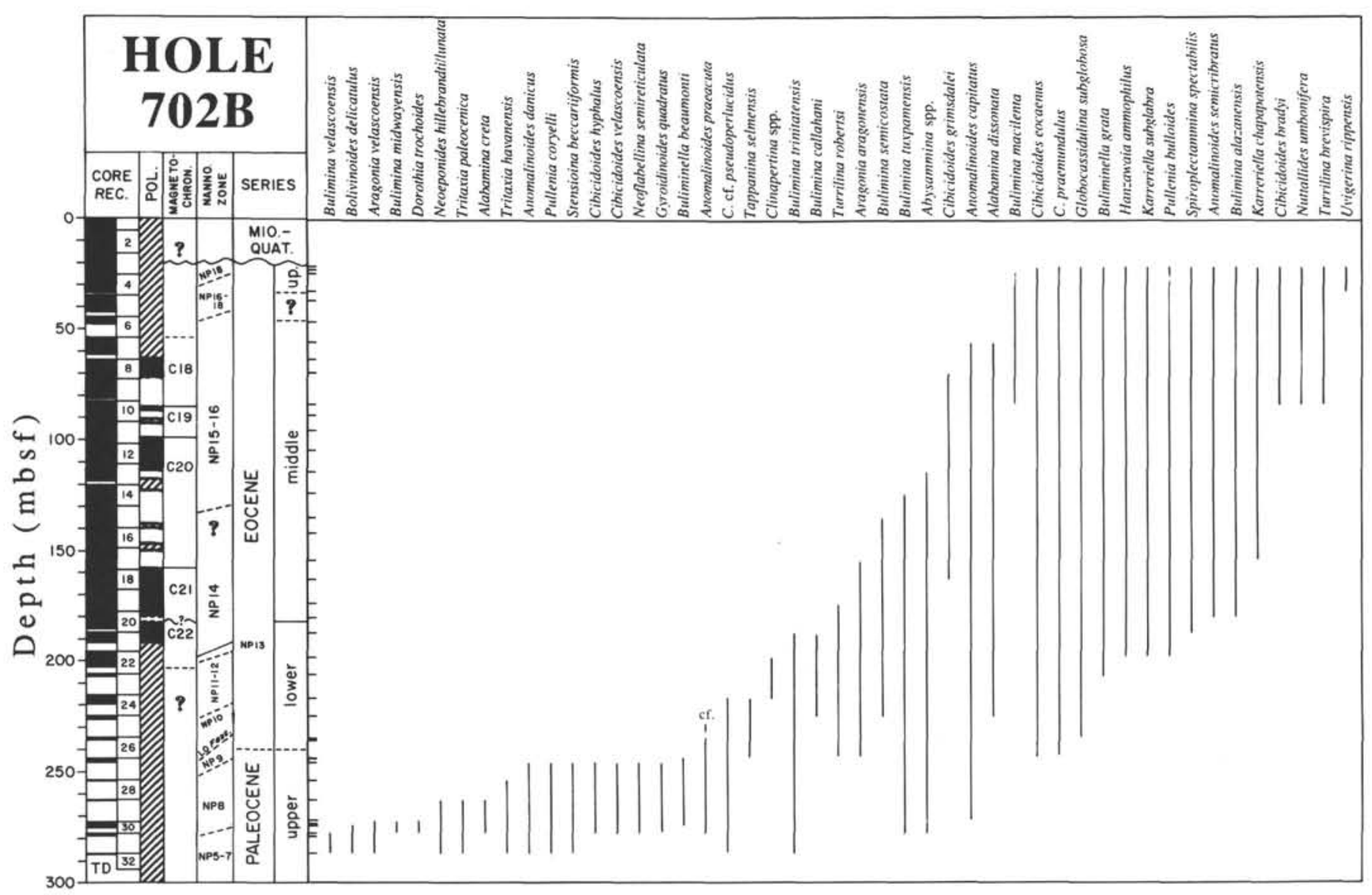

Figure 6. Ranges of benthic foraminifers at Hole 702B. Dashed line $=$ uncertain or possible range; tick marks indicate samples examined for benthic foraminiferal stratigraphic ranges. LO Fasc. = last occurrence of the nannofossil Fasciculithus spp. Nannofossil biostratigraphy and magnetostratigraphy after Shipboard Scientific Party (1988d). Normal polarity is indicated in black; reversed polarity is in white. Ruled pattern indicates intervals of indeterminate magnetostratigraphy.

Paleocene section shows obvious optical evidence of recrystallization. We believe that the oxygen isotope record of the Paleocene has been altered at this location. Oxygen isotope values from this section are anomalously low (i.e., much lower than coeval records), although $\delta^{13} \mathrm{C}$ values appear unaltered. However, the Eocene section at Site 700 yielded well-preserved benthic foraminifers across a $175 \mathrm{~m}$ interval.

Shackleton et al. (1984) measured paired samples of Cibicidoides and $N$. truempyi and concluded that Cibicidoides were lower in $\delta^{18} \mathrm{O}$ by approximately $0.15 \%$ but similar in $\delta^{13} \mathrm{C}$ composition to $N$. truempyi. We compared paired analyses of Cibicidoides and $N$. truempyi from Shackleton et al. (1984) with paired analyses from Sites 384 (Miller et al., 1987c), 700, and 702. We found (Fig. 14) that $N$. truempyi was depleted in ${ }^{18} \mathrm{O}$ by $0.1 \%$ relative to Cibicidoides spp. In addition, our data suggest that there is a systematic offset between Cibicidoides and $N$. truempyi in $\delta^{13} \mathrm{C}$ composition, with the latter being depleted by $0.26 \%$ (Fig. 14). Still, there is considerable scatter in these comparisons and more data are needed to determine both the precise offset and its variation through time.

Oxygen isotope values at Hole $702 \mathrm{~B}$ decrease by $0.9 \%$ in the uppermost Paleocene to lower Eocene (Fig. 15). The decrease may have begun as far below the Paleocene/Eocene boundary as Core 114-702B-29X (approximately $59.5 \mathrm{Ma}$ ); a sharp decrease of approximately $0.3 \%$ occurs within Core 114-702B-26X (approximately $235 \mathrm{mbsf}$; approximately $57 \mathrm{Ma}$ )
(Fig. 15). Oxygen isotope values attain a distinct minimum $(0.8 \%$ in Cibicidoides) in the lower Eocene (approximately 200 mbsf; approximately $53.7 \mathrm{Ma}$ ) (Fig. 15). If the entire signal is ascribed to temperature change, this decrease across the $\mathrm{Pa}$ leocene/Eocene boundary represents a deep-water warming of about $4^{\circ} \mathrm{C}$ from approximately 57 to $54 \mathrm{Ma}$. The minimum $\delta^{18} \mathrm{O}$ values correspond to deep-water temperatures of $12^{\circ} \mathrm{C}$ (using the paleotemperature equation $\delta_{w}=-1.2 \%$ of O'Neil et al., 1969; Shackleton and Kennett, 1975).

The oxygen isotope values increase at Hole 702B by $1.2 \%$ from the upper lower Eocene to the lower middle Eocene (190 to $152 \mathrm{mbsf}$ ) (Fig. 15). This increase is the first step of a general middle Eocene to Oligocene $\delta^{18} \mathrm{O}$ increase. At Site 702, this increase begins in Chronozone C22N (52.6$52 \mathrm{Ma}$ ) and continues up into Chronozone $\mathrm{C} 21 \mathrm{~N}$ (approximately $49 \mathrm{Ma}$ ). The best previous record constraining this $\delta^{18} \mathrm{O}$ increase was reported by Shackleton et al. (1984) from South Atlantic DSDP Site 527 . At that site, the $\delta^{18} \mathrm{O}$ increase apparently began in Chronozone $\mathrm{C} 21 \mathrm{~N}$ (lowermost middle Eocene; 50.34-48.75 Ma); however, there was poor recovery and no stable isotope data were available for Chronozone C22 at DSDP Site 527. At Pacific DSDP Site 577, the oxygen isotope increase apparently begins in normally magnetized sediments assigned to Chronozone $\mathrm{C} 22 \mathrm{~N}$, although only three points constrain this because there is an unconformity at the top of Chronozone C22N (Miller et al., 1987c). Thus, our record at Site 702 provides the first complete estimate of the 


\section{FACTOR 3}

FACTOR 1

FACTOR 4

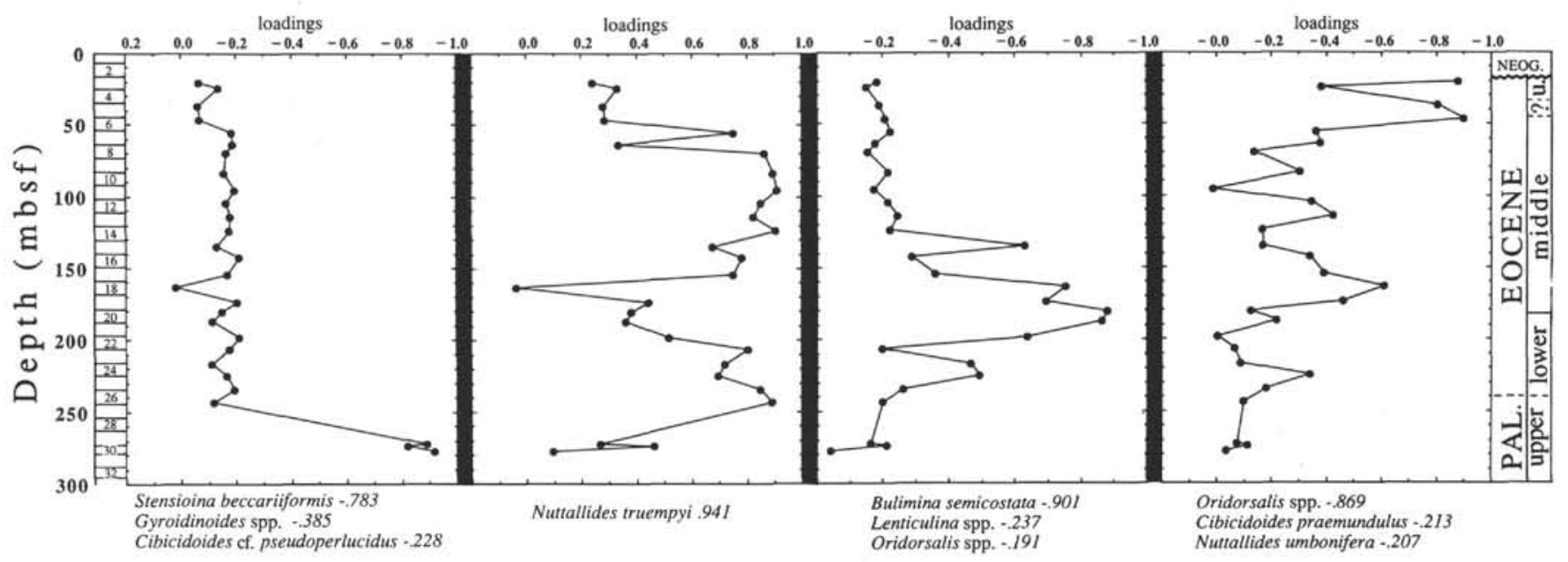

Figure 7. Q-mode Varimax factor analysis of benthic foraminiferal relative abundance data from Hole 702B. Factors are arranged in stratigraphic sequence of successive assemblages. Core numbers are to the left. Species contributing to each factor and their factor scores are indicated under each column. Factor 1 explains $41 \%$ of the variance, factor 2 explains $15 \%$ of the variance, factor 3 explains $10 \%$ of the variance, and factor 4 explains $17 \%$ of the variance.

timing of the oxygen isotope increase: it began in the latest early Eocene (approximately 52.6-52.0 Ma) and continued into the early middle Eocene (49 Ma). The amplitude of the event makes it one of the largest $\delta^{18} \mathrm{O}$ increases of the Cenozoic.

An $0.8 \delta^{18} \mathrm{O}$ increase occurs near the middle/upper Eocene boundary at Hole 702B. The timing of this increase cannot be well constrained here or elsewhere. Keigwin and Corliss (1986) reported a $1 \%$ increase in benthic foraminiferal $\delta^{18} \mathrm{O}$ values at two South Atlantic locations (DSDP Sites 19 and 363); at these sites the increase begins in the upper middle Eocene and apparently extends into the upper Eocene. Oberhansli et al. (1984) reported an approximately $0.8 \%$ oo increase in benthic foraminiferal $\delta^{18} \mathrm{O}$ values from South Atlantic Site 523 in the upper middle Eocene. At Hole 702B, the increase occurs between Samples 114-702B-8X-3, 50-54 cm, and 114702B-6X-2, 100-104 cm (66.8-46.8 mbsf) (Table 2 and Fig. $15)$. We estimate that the increase occurred in the latest middle Eocene (approximately 42-41 Ma), although the chronology of this part of the Hole 702B section is unclear. The increase begins within Chronozone C18N (42.73-41.29 Ma) and occurs mostly within the range of Acarinina primitiva (LO at 47.50 to 46.07 mbsf; P. F. Ciesielski, unpubl. data; i.e., prior to the LO of Acarinina spp., $40.6 \mathrm{Ma}$, Berggren et al., 1985). Therefore, the $\delta^{18} \mathrm{O}$ increase is assignable to the late middle Eocene (approximately 42-40 Ma). However, the poor recovery across this interval precludes definite correlation of the $\delta^{18} \mathrm{O}$ increase to the time scale. The increase begins in uppermost middle Eocene sediments at Sites 19, 363, 523, and 363 , suggesting that this was a synchronous change, although the data do not allow precise correlation.

The early to middle Eocene and late middle Eocene $\delta^{18} \mathrm{O}$ events represent nearly $2.0 \%$ of cumulative increase; thus, one of the largest climatic transitions of the Cenozoic occurred during the middle Eocene. Both increases are similar in amplitude to the widely known earliest Oligocene $\delta^{18} \mathrm{O}$ increase of $\sim 1.0 \%$. Our records suggest that the two middle Eocene $\delta^{18} \mathrm{O}$ increases each occurred over several million years and were not as rapid as the earliest Oligocene $\delta^{18} \mathrm{O}$ increase. Data from Leg 113 confirm this (Kennett and Stott, 1990).
As a result of this recrystallization and poor recovery at Hole 700B, the $\delta^{18} \mathrm{O}$ decrease (Fig. 16), which occurred elsewhere near the Paleocene/Eocene boundary, is not documented. However, a $\delta^{18} \mathrm{O}$ increase of about $1.3 \%$ is recorded across the lower/middle Eocene boundary into the middle middle Eocene at Hole 700B (Fig. 16). This increase is therefore coeval with the increase noted at Hole 702B.

The oxygen isotope records afforded by Sites 698 and 699 are not complete time series (Table 2). However, the values obtained are consistent with those obtained from the more complete Sites 700 and 702 (Fig. 17). The Paleocene to lowermost Eocene of Site 699 suffers from diagenetic recrystallization like that at Site 700 (Fig. 17). With the exception of these two altered upper Paleocene sections, comparison of the $\delta^{18} \mathrm{O}$ records from all four locations shows that coeval $\delta^{18} \mathrm{O}$ values are similar among the locations, supporting the stratigraphic correlation of the records.

The sections at Holes $700 \mathrm{~B}$ and 702B also record large decreases in benthic foraminiferal carbon isotope values beginning in the upper Paleocene and culminating in the lower Eocene (Figs. 15 and 16 ). Hole $700 \mathrm{~B} \delta^{13} \mathrm{C}$ values decrease by about $2.5 \%$ oo-2.9\% between 249 and 196 mbsf (Fig. 16), while the $\delta^{13} \mathrm{C}$ values at Hole 702B decrease by about $2.4 \%$ between 273 and 219 mbsf (Fig. 15). This carbon isotope decrease noted at Holes $700 \mathrm{~B}$ and $702 \mathrm{~B}$ is global in extent (Shackleton and Hall, 1984; Shackleton et al., 1984; Shackleton, 1987; Miller et al., 1987c; Kennett and Stott, 1990).

Although the upper Paleocene oxygen isotope record from Hole $700 \mathrm{~B}$ is altered (with coeval values significantly lower than at Hole 702B), synoptic carbon isotope values appear similar between Holes 700B and 702B. The carbon isotope values from the Paleocene of Hole 700B are not altered to the extent of the oxygen isotope values; Keigwin and Corliss (1986) noted similarly that sections with altered oxygen isotope records had essentially unaltered carbon isotope values due to the lower temperature coefficients for carbon isotopes and the smaller reservoir of carbon in pore waters. We therefore plot and interpret the carbon isotope records from the Paleocene of Holes 699 and 700 (Figs. 18 and 19) despite the alteration of the oxygen isotope records from these sections. 


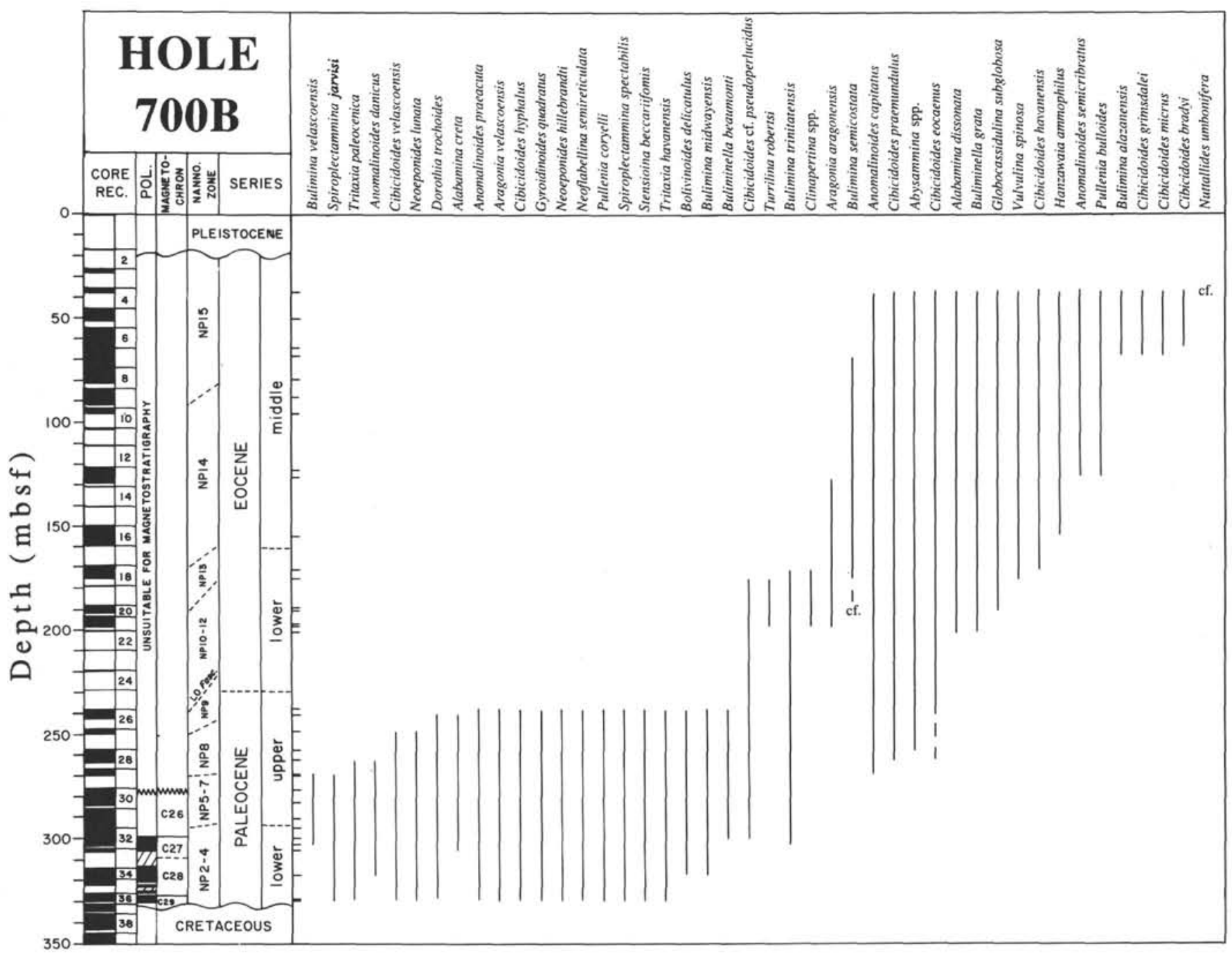

Figure 8. Ranges of benthic foraminifers at Hole 700B. Dashed line = uncertain or possible range; tick marks indicate samples examined for benthic foraminiferal stratigraphic ranges. LO Fasc. = last occurrence of the nannofossil Fasciculithus spp. Nannofossil biostratigraphy and magnetostratigraphy after Shipboard Scientific Party (1988c). Normal polarity is indicated in black; reversed polarity is in white. Ruled pattern indicates intervals of indeterminate magnetostratigraphy.

There are few changes in $\delta^{13} \mathrm{C}$ values in the middle Eocene at either Hole $700 \mathrm{~B}$ or Hole $702 \mathrm{~B}$ (Figs. 15 and 16), with relatively constant values throughout the sections $(1.0 \%)$. We cannot evaluate if this is a global or local pattern, because there is no record for comparison with the middle Eocene $\delta^{13} \mathrm{C}$ composition of the Pacific Ocean, which forms the bulk of the oceanic reservoir. Because deep-water reconstructions require constraints from Pacific $\delta^{13} \mathrm{C}$ records, we focus our efforts here on the late Paleocene to early Eocene interval.

Comparisons of Pacific and Southern Ocean oxygen isotope records reveal intriguing possibilities for deep-water circulation changes in the Paleogene. Prior to $60 \mathrm{Ma}$, Southern Ocean locations had distinctly lower $\delta^{18} \mathrm{O}$ values relative to Pacific Site 577 (approximately $1950 \mathrm{~m}$ paleodepth) and deepwater temperatures are inferred to have been warmer than those of the Pacific (Fig. 20; fig. 6 in Miller et al., 1987c). From approximately 60 to $58 \mathrm{Ma}, \delta^{18} \mathrm{O}$ records at Southern Ocean Sites 702 and 524 became similar to or more enriched than the record at Pacific Site 577 (Fig. 20; fig. 6 in Miller et al., 1987c); this suggests that Southern Ocean deep-water temperatures were similar to or colder than the Pacific temperatures. The differences observed are larger than the potential differences due to species effects (i.e., the Site 702 record in Fig. 20 is based on Cibicidoides, whereas the records from Sites 524 and 577 are of Nuttallides truempyi). These $\delta^{18} \mathrm{O}$ differences are a consequence of the fact that the oxygen isotope decrease (deep-water warming) began at Pacific Site 577 around $61 \mathrm{Ma}$, but did not begin in the Southern Ocean until about 59 or 58 Ma (fig. 6 in Miller et al., 1987c), and continued across the Paleocene/Eocene boundary. Hence, water with a higher $\delta^{18} \mathrm{O}$ signature (i.e., relatively cooler) began to enter the Southern Ocean in the late Paleocene even though global deep waters warmed from about 61 to $54 \mathrm{Ma}$. This is consistent with the supply of relatively cooler (albeit warm) high-latitude waters to the Southern Ocean during the late Paleocene to early Eocene.

Carbon isotope comparisons confirm our suggestion that nutrient-depleted deep water filled the Atlantic sector of the Southern Ocean during the late Paleocene (approximately $60-58 \mathrm{Ma}$ ). Prior to approximately $60 \mathrm{Ma}$ there seems to have been little $\delta^{13} \mathrm{C}$ difference between the Southern Ocean (Sites 524, 700, and 702) and Pacific (Site 577) locations (Figs. 20 and 
FACTOR 2

FACTOR 3

FACTOR 1

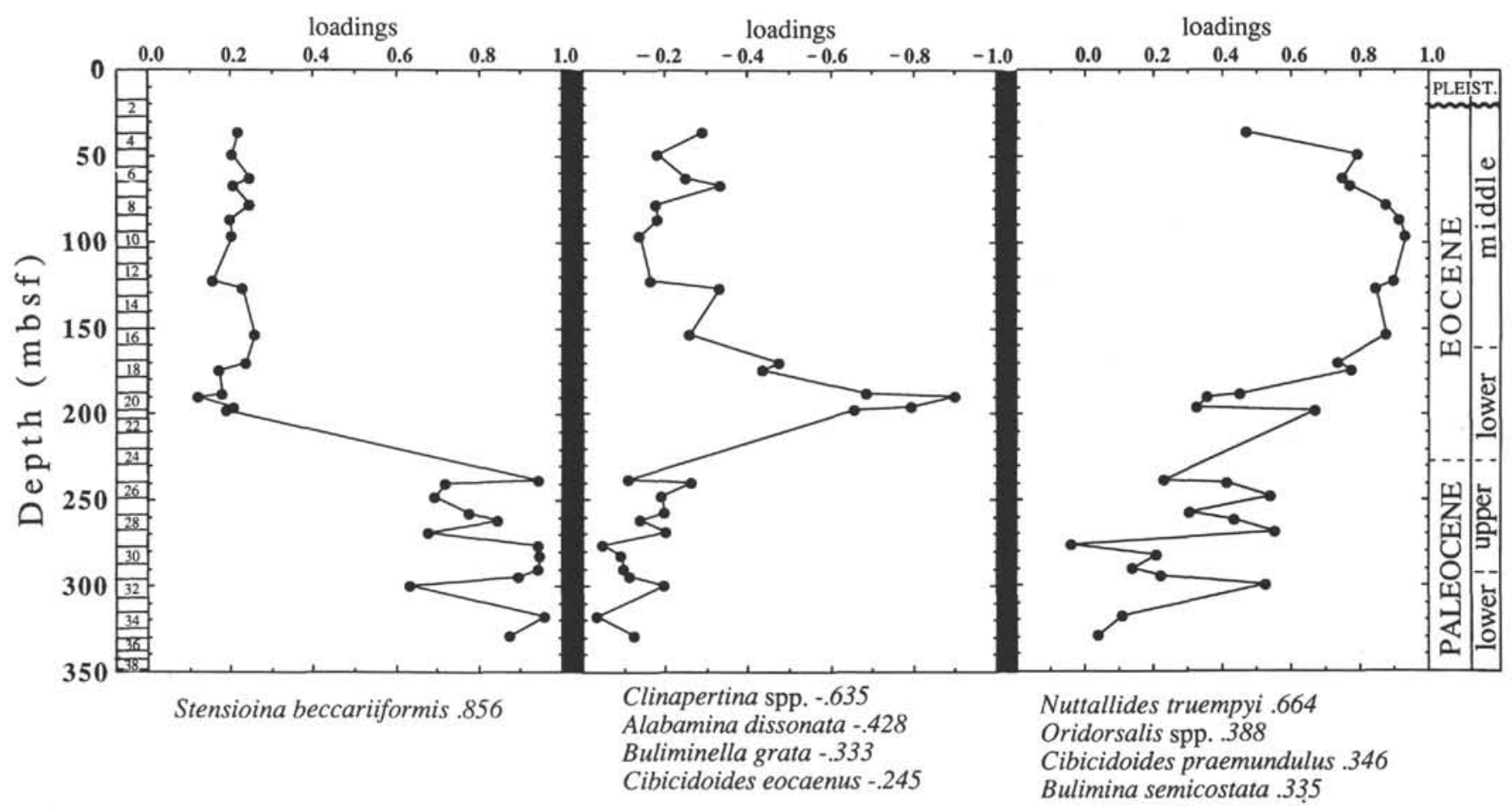

Figure 9. Q-mode Varimax factor analysis of benthic foraminiferal relative abundance data from Hole 700B. Factors are arranged in stratigraphic sequence of successive assemblages. Core numbers are to the left. Species contributing to each factor and their factor scores are indicated under each column. Factor 1 explains $35 \%$ of the variance, factor 2 explains $34 \%$ of the variance, and factor 3 explains $12 \%$ of the variance.

21). The western basins of the Atlantic (Sites 356, 357, 98, and 384; Brazil and American basins) were also similar to those of the Pacific during the interval $63-61 \mathrm{Ma}$, and the oceans appear to have had a fairly uniform $\delta^{13} \mathrm{C}$ composition prior to $61 \mathrm{Ma}$ (Fig. 21).

Beginning at about $60 \mathrm{Ma}$, the Southern Ocean locations were enriched in $\delta^{13} \mathrm{C}$ by more than $0.5 \%$ relative to the Pacific (Figs. 20 and 21). We did not adjust either the oxygen or carbon isotope values on Figure 20 or Figure 21; if $N$. truempyi is enriched in carbon by $0.26 \%$ relative to Cibicidoides (see preceding discussion), then the difference from the Southern Ocean to the Pacific would have been less (i.e., the Pacific Site 577 record is from N. truempyi). Still, the available N. truempyi data from the Southern Ocean (plotted as $\times$ 's in Fig. 20; the entire Site 524 record in Fig. 21) are enriched relative to the Pacific. Limited data from the western basins of the Atlantic (Sites 20, 21, 98, and 144) suggest that these locations remained similar to the Pacific in $\delta^{13} \mathrm{C}$ composition during most of the latest Paleocene (approximately $60-58 \mathrm{Ma}$ ) (Fig. 21). The Southern Ocean-Pacific $\delta^{13} \mathrm{C}$ difference may have been reduced or eliminated between 58 and $57 \mathrm{Ma}$ (across the Paleocene/Eocene boundary) (Figs. 20 and 21). During the early Eocene, Leg 114 Sites 700 and 702 were enriched in ${ }^{13} \mathrm{C}$ by up to $1 \%$ relative to the Pacific, indicating a high supply of nutrient-depleted deep water to this region of the ocean (Figs. 20 and 21). The early Eocene record from the Cape Basin Site 524 is similar to that from the Pacific; however, this is based upon only two data points. Therefore, it is not clear if the entire Atlantic sector of the Southern Ocean was flooded with ${ }^{13} \mathrm{C}$-enriched deep water during the early Eocene.

Age-paleodepth reconstructions of the carbon isotope distribution for the Southern Ocean region near the Falkland
Islands show that for much of the late Paleocene to early Eocene this region was vertically well mixed below approximately $800 \mathrm{~m}$ with respect to $\delta^{13} \mathrm{C}$ (Fig. 18). The exception is near 54-53 Ma, when there appears to have been a small vertical gradient (Fig. 18); carbon isotope values at Site 702 were slightly lower from 54 to $53 \mathrm{Ma}$ than they were at Sites 698, 699, and 700 (Fig. 18).

The distribution of carbon isotopes in the Southern Ocean (Fig. 18) alone does not constrain deep-water circulation history; the global carbon isotope signal must be removed to reveal the deep-water circulation signal. We did this by using Pacific Site 577 as an approximation for the deep-water of the Pacific and subtracting from it our Southern Ocean records to show that the region east of the Falklands was enriched relative to the Pacific by $0.5 \% 0-1.0 \%$ in the late Paleocene (approximately 60-58 Ma) and early Eocene (approximately 57-54 Ma) (Fig. 19A). There apparently was a smaller difference $(<0.5 \%)$ near the Paleocene/Eocene boundary $(58-57$ Ma). Our reconstruction confirms what can be seen in the comparisons of individual time series (Figs. 20 and 21) and demonstrates that the Southern Ocean was enriched in ${ }^{13} \mathrm{C}$ relative to the Pacific over a wide bathymetric range.

To test the sensitivity of Figure $19 \mathrm{~A}$ to potential species effects, we adjusted the $N$. truempyi values used to construct Figure $19 \mathrm{~B}$ by adding $0.26 \%$, which is the difference between $N$. truempyi and Cibicidoides suggested by Figure 14; this conservative reconstruction de-emphasizes differences between basins. The age-paleodepth compilation (Fig. 19B) shows that the Southern Ocean locations were enriched in ${ }^{13} \mathrm{C}$ relative to the Pacific for much of the late Paleocene to early Eocene. However, there are intervals when the Southern Ocean appears depleted in ${ }^{13} \mathrm{C}$ relative to the Pacific. For example, near the Paleocene/Eocene boundary, $\delta^{13} \mathrm{C}$ values in 


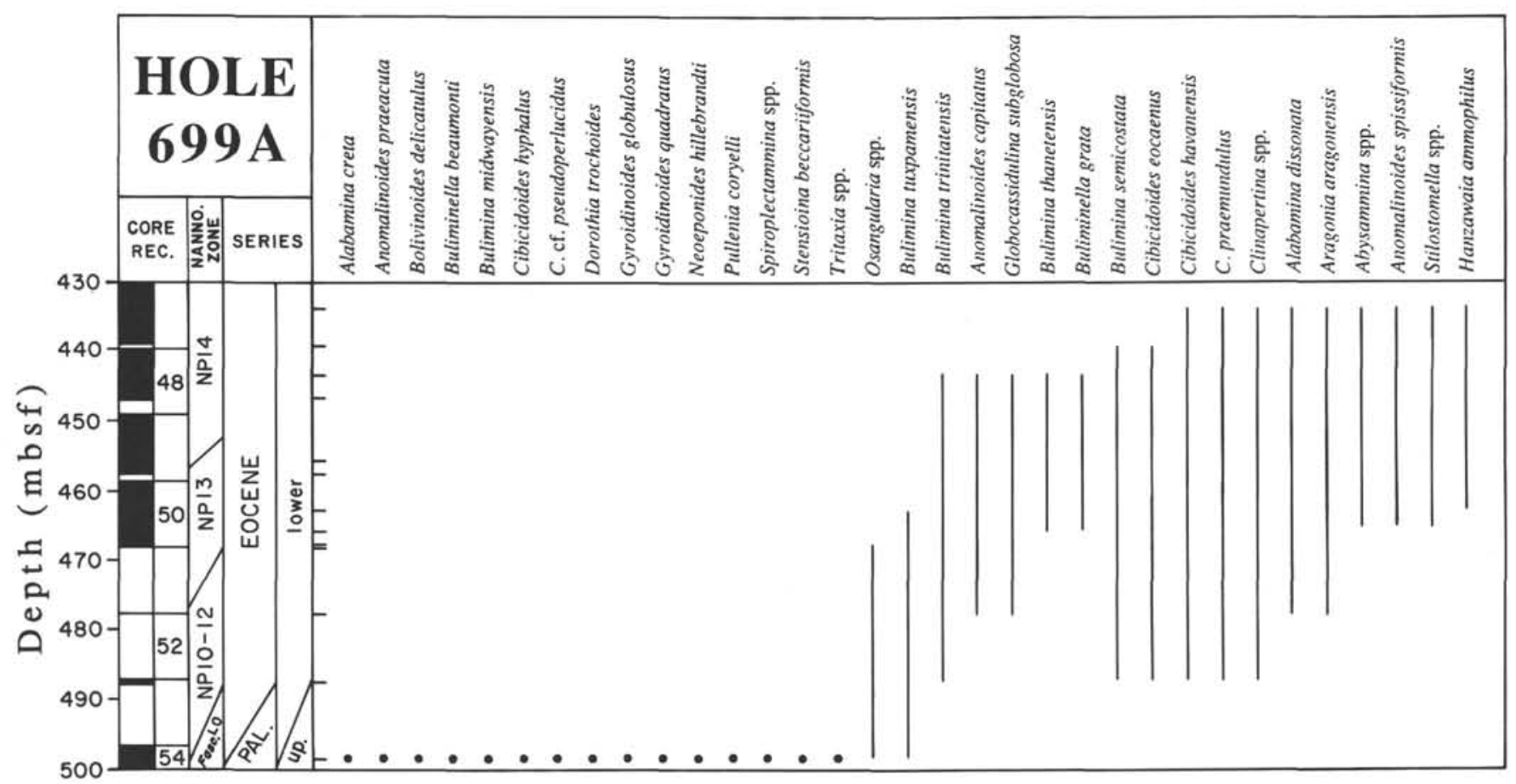

Figure 10. Ranges of benthic foraminifers at Hole 699A. Solid circles = more than one specimen of a taxon that is present in only one sample; tick marks indicate samples examined for benthic foraminiferal stratigraphic ranges. LO Fasc. = last occurrence of the nannofossil Fasciculithus spp. Nannofossil biostratigraphy after Shipboard Scientific Party (1988b).

the Southern Ocean (at depths $<1800 \mathrm{~m}$ ) were lower than those in the Pacific. If this reconstruction is correct, this suggests no supply of ${ }^{13} \mathrm{C}$-enriched water to this level of the Southern Ocean near the Paleocene/Eocene boundary.

Both reconstructions (Figs. 19A and 19B) show that there were (1) large $\delta^{13} \mathrm{C}$ differences between the Pacific Ocean and the Southern Ocean during the late Paleocene and early Eocene and (2) a reduction or elimination of supply of "young" deep water to the Southern Ocean near the $\mathrm{Pa}$ leocene/Eocene boundary. Data from Sites 689 and 690 on the Maud Rise (Kennett and Stott, 1990) also show large $\delta^{13} \mathrm{C}$ differences between the Pacific and Southern Ocean during the late Paleocene and early Eocene. Their isotope records are more detailed near the Paleocene/Eocene boundary, and they show a large transient $\delta^{13} \mathrm{C}$ decrease $(<0.5 \mathrm{~m} . \mathrm{y}$.) in the Southern Ocean at this time. This is consistent with elimination of "young" bottom water in the Southern Ocean near the Paleocene/Eocene boundary; alternatively, the transient $\delta^{13} \mathrm{C}$ decrease may reflect a global carbon budget change. Additional detailed Pacific $\delta^{13} \mathrm{C}$ records are needed to distinguish between these two scenarios.

\section{DISCUSSION}

\section{Sources of Early Paleogene Deep Water}

Our reconstructions firmly document that the Atlantic sector of the Southern Ocean was enriched in ${ }^{13} \mathrm{C}$ relative to the Pacific during much of the late Paleocene (approximately 60-58 Ma) over a wide bathymetric range (Sites 524, 698, 699, 700 , and $702 ; \sim 800-3400 \mathrm{~m}$ ) (Figs. 19-21); in addition, the sites east of the Falkland Islands (Sites 698, 699, 700, and 702; $\sim 800-3000 \mathrm{~m}$ paleodepth) were enriched in ${ }^{13} \mathrm{C}$ relative to the Pacific during the early Eocene (approximately 57-52 Ma). There is evidence for "old" nutrient-enriched deep water in the western north Atlantic (Site 98) (Fig. 21), suggesting that there was no North Atlantic source for much of this interval. Hence, the nutrient-depleted deep water may have come from the antarctic region, a source we favor, or it may have been supplied from the Tethys. Warm, high-salinity Tethyan deep water analogous to the warm saline bottom water of Brass et al. (1982) and Kennett and Stott $(1988,1990)$ may have entered the Southern Ocean via the Indian Ocean; if this Tethyan water was high in oxygen and stripped of nutrients (resulting in high $\delta^{13} \mathrm{C}$ values), it potentially may have served as the source for the "young" nutrient-depleted deep water found in the Southern Ocean.

We believe that the antarctic region is the most likely source for the late Paleocene and Eocene nutrient-depleted deep water. The Southern Ocean changed from relatively warmer (compared with the Pacific) deep-water temperatures to relatively colder deep-water temperatures at the same time as it became enriched in ${ }^{13} \mathrm{C}$ relative to the Pacific. This correspondence is further supported by the fact that the highest late Paleocene benthic foraminiferal $\delta^{13} \mathrm{C}$ values reported are from the Southern Ocean. Stable isotope records from closer to the potential source regions (eastern Tethys or Weddell Sea) should allow this nutrient-depleted deep water to be traced back to its source.

\section{Timing and Cause of the Benthic Foraminiferal Crisis}

While the nature of the late Paleocene benthic foraminiferal crisis has been well documented, the precise timing of the benthic foraminiferal extinction event near the Paleocene/ Eocene boundary has remained elusive. Tjalsma and Lohmann (1983) observed that characteristic Paleocene benthic foraminiferal taxa present in their Zone P5 samples were absent from their Zone P6a samples, suggesting that the final extinction event occurred between these levels. However, the benthic foraminiferal taxonomic record at the Pacific Site 577 shows that the extinction event occurred between samples 


\section{Principal Component 2}

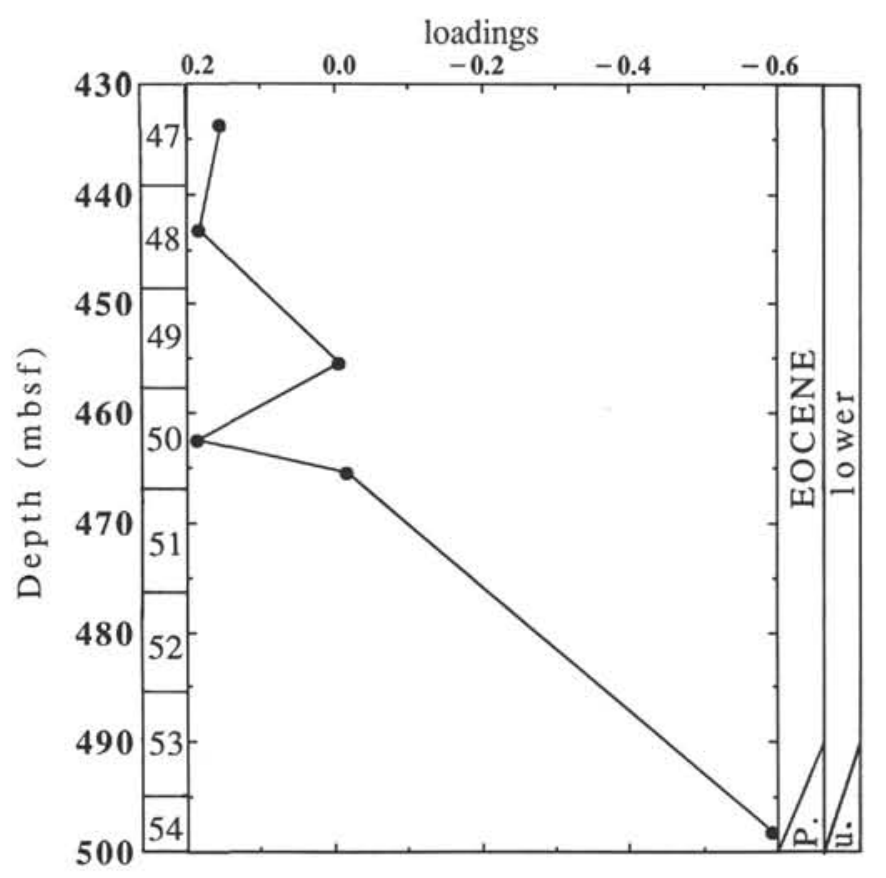

Cibicidoides cf. pseudoperlucidus -.395

Cibicidoides hyphalus -.344

Stensioina beccariiformis -.275

Figure 11. Q-mode principal component analysis of benthic foraminiferal relative abundance data from Hole 699A. Core numbers are to the left. Principal component scores are given for species contributing to the second principal component. Principal component 2 explains $23 \%$ of the variance.

examined from Zones P6a and P6b (Miller et al., 1987c). The benthic foraminiferal turnover clearly spans the Paleocene/ Eocene transition at our sites. Unfortunately, the biostratigraphic age control is insufficient at the Leg 114 sites examined in this study to clarify the precise timing of the Paleocene/ Eocene event further. However, timing of the change is constrained at Site 690 , where it clearly predates the $\mathrm{Pa}$ leocene/Eocene boundary (age estimate slightly younger than $58 \mathrm{Ma}$; Thomas, 1990, in press). The timing of the change at our sites is consistent with the estimate of Thomas (1990, in press) for the more complete section at Site 690 .

The cause of the faunal turnover is still undetermined. It occurs during the general warming of deep water between approximately 60 and $56 \mathrm{Ma}$. Still, temperature change alone may not have been the cause of the extinctions. Comparison of the oxygen and carbon isotope records at Sites 577 and 702 with the faunal turnover at these sites (Fig. 20) shows that (1) the turnover in the Pacific (Site 577) postdates the largest inflection in the oxygen isotope record, while (2) the extinctions at Site 702 occurred before the largest inflection in the $\delta^{18} \mathrm{O}$ record. It is not clear if these sharp inflections are real at these sites, for they are constrained only by single data points (Fig. 20). Still, the relationship between the extinctions and the $\delta^{18} \mathrm{O}$ record is not convincing from the available data (Fig. 20).

Nor can the faunal turnover be directly attributed to a drop in food supply. The bulk of the extinctions occurred during the large carbon isotope decrease. Even if this $\delta^{13} \mathrm{C}$ decrease is attributed to a drop in surface ocean productivity (Shackleton et al., 1985b) and food supply to the benthos, there appears no direct link between the extinctions and the carbon isotope change (Fig. 20). One possible cause of the turnover is a change in the source region for deep waters (Miller et al., $1987 \mathrm{c}$; Thomas, 1988, 1990, in press). Our comparisons with the Pacific clearly show that the $\delta^{13} \mathrm{C}$ difference with the Southern Ocean was reduced or eliminated near the Paleocene/Eocene boundary (58-57 Ma) (Figs. 19-21). Following this, the difference between basins was reestablished (Figs. 19-21). We speculate that the Southern Ocean provided a deep-water source during the late Paleocene (approximately 60-58 Ma). This Southern Ocean source was eliminated near the Paleocene/Eocene boundary and was replaced by another deep-water source, possibly a low-latitude supply (e.g., Thomas, 1988,1990 , in press). This change in source regions may have caused the extinctions of the benthic foraminifers. Commencing in the early Eocene (approximately $57 \mathrm{Ma}$ ), the Southern Ocean source was again renewed, and the benthic foraminifers radiated to fill the vacated niches.

Faunal abundance changes are consistent with this scenario. The greatest difference between the Pacific and Southern Ocean $\delta^{13} \mathrm{C}$ records occurred between approximately 60 and $59 \mathrm{Ma}$ (Figs. 19-21). The difference began to decrease between 59 and $58 \mathrm{Ma}$, and was virtually eliminated from 58 to $57 \mathrm{Ma}$. The abundance of Stensioina beccariiformis began to drop in the Pacific at about $59 \mathrm{Ma}$ (Fig. 13); it lingered on in higher abundances in the Southern Ocean until approximately 58 Ma (Figs. 12 and 13). Similarly, the demise of the $S$. beccariiformis-dominated assemblage may have begun earlier in the Atlantic-Caribbean than in the Southern Ocean (Tjalsma and Lohmann, 1983). We speculate that reduced supply of Southern Ocean deep water beginning at approximately $59 \mathrm{Ma}$ resulted in the decrease in the $S$. beccariiformis-dominated assemblage in the more peripheral regions (Pacific and Atlantic-Caribbean); only with the virtual shutdown of deep-water production in this region near the Paleocene/Eocene boundary (58-57 Ma) was the $S$. beccariiformis assemblage eliminated in the region proximal to the deep-water source, the Southern Ocean.

\section{CONCLUSIONS}

The benthic foraminiferal extinction event documented in the Atlantic and Pacific oceans culminated near the Paleocene/ Eocene boundary (approximately 58-57 Ma) at Southern Ocean Sites 698, 699, 700, and 702, spanning paleodepths of about $800-3000 \mathrm{~m}$. An assemblage dominated by Stensioina beccariiformis persisted in these Southern Ocean locations until near the end of the Paleocene. It was replaced by a Nuttallides truempyi-dominated assemblage. The late Paleocene of the Southern Ocean was remarkably uniform in its faunal and stable isotope composition. In contrast, several depth-related benthic foraminiferal distributions developed in the Eocene Southern Ocean.

The pieces of the Paleocene/Eocene extinction puzzle are not complete despite the data assembled by ODP Legs 113 and 114. Still, of the three competing hypotheses for the cause(s) of the extinctions (temperature increase, productivity decrease, or change in deep-water source regions), our data are most consistent with a change in source regions near the Paleocene/Eocene boundary.

Stable isotope results from the lower Paleogene sections recovered by Leg 114 show that:

1. Deep and bottom waters were warmer in the Southern Ocean than in the Pacific prior to $60 \mathrm{Ma}$. The amplitude of the $\delta^{18} \mathrm{O}$ decrease across Paleocene/Eocene boundary was less in the Southern Ocean than in the Pacific; hence, relatively 


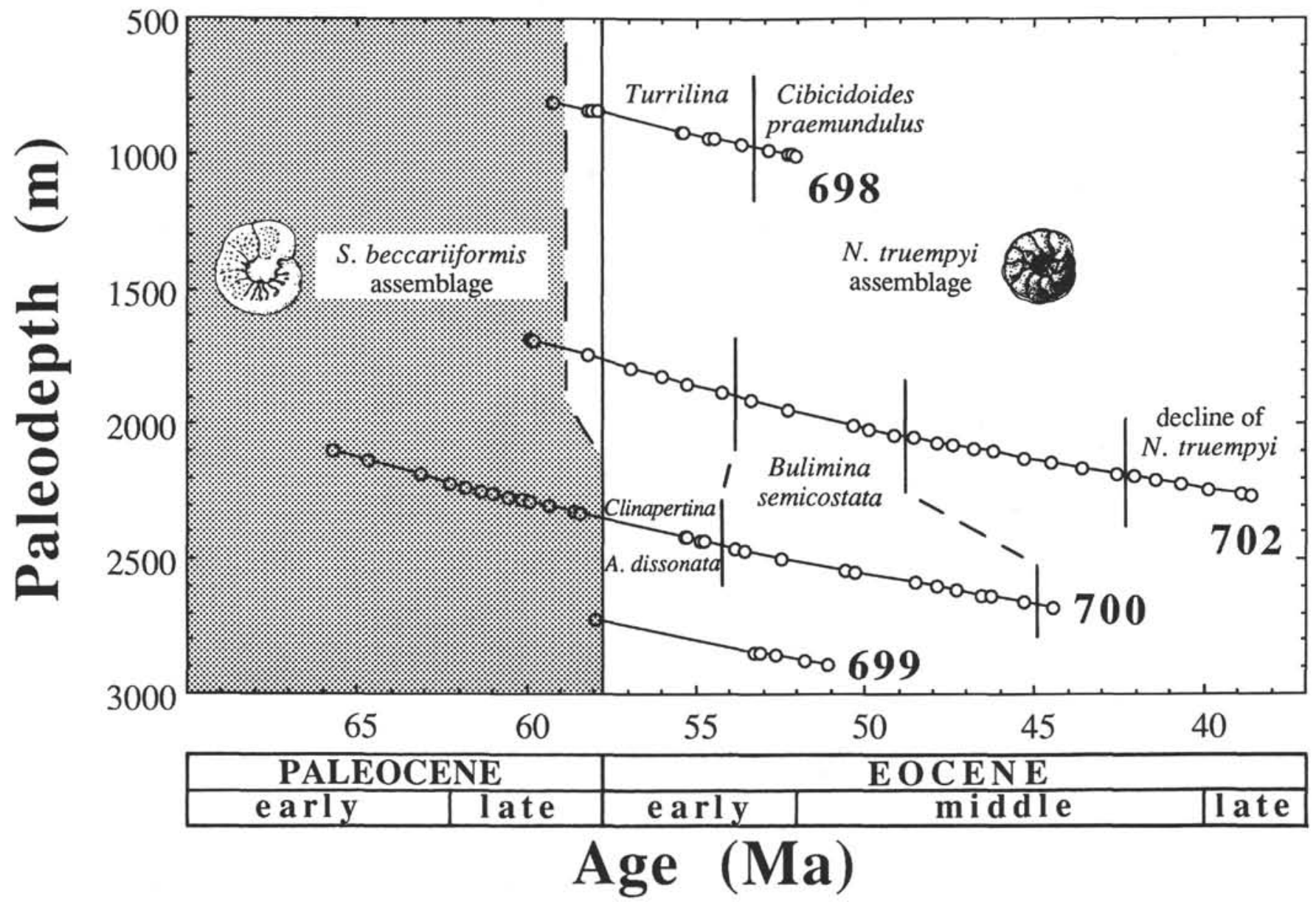

Figure 12. Age-paleodepth reconstruction of Paleocene-Eocene benthic foraminiferal assemblages in the Southern Ocean for Leg 114 . Illustrations after Tjalsma and Lohmann (1983).

cooler water entered this part of the Southern Ocean even though global deep waters warmed from $\sim 61-54 \mathrm{Ma}$.

2. Major benthic foraminiferal $\delta^{18} \mathrm{O}$ increases occurred in the latest early to early middle Eocene (Chronozones $\mathrm{C} 22 \mathrm{~N}-$ $\mathrm{C} 20 \mathrm{~N}$; approximately $52.6-49 \mathrm{Ma})$ and in the latest middle Eocene (approximately $42-41 \mathrm{Ma}$ ).

3. Southern Ocean benthic foraminiferal $\delta^{13} \mathrm{C}$ records are enriched relative to the Pacific in the late Paleocene (approximately 60-58 Ma) and in the early Eocene (approximately 57-52 Ma). There appears to have been less of a difference between the Southern Ocean and Pacific $\delta^{13} \mathrm{C}$ records in the vicinity of the Paleocene/Eocene boundary (58-57 Ma).

We interpret the carbon and oxygen isotope records as reflecting deep-water production in the region near the Atlantic sector of the Southern Ocean from approximately 60 to $58 \mathrm{Ma}$ and from 57 to $52 \mathrm{Ma}$. We speculate that reduction or elimination of the Southern Ocean source near the Paleocene/Eocene boundary (58-57 Ma) may have been the primary cause of the benthic foraminiferal extinction event.

\section{ACKNOWLEDGMENTS}

We thank S. Savin and E. Thomas for reviews, R. C. Tjalsma for discussions of benthic foraminiferal taxonomy, E. Thomas for discussions of the Paleocene/Eocene turnover, D. Greig (Chevron) for producing the SEM micrographs, and $\mathrm{N}$. Katz for drafting. Samples were provided by ODP. This work was supported by JOI/USSAC and National Science Founda- tion grant OCE88-17563. This is an Lamont-Doherty Geological Observatory Contribution.

\section{REFERENCES}

Aubry, M.-P., Berggren, W. A., Kent, D. V., Flynn, J. J., Klitgord, K. D., Obradovich, J. D., and Prothero, D. R., 1988. Paleogene geochronology: an integrated approach. Paleoceanography, 3:707-742.

Barrera, E., Huber, B. T., Savin, S. M., and Webb, P.-N., 1987. Antarctic marine temperatures: late Campanian through early Paleocene. Paleoceanography, 2:21-47.

Barron, J. A., Larsen, B., Baldauf, J. G., et al., 1988. Development of the East Antarctic Ice Sheet and related paleoceanographic changes: results from ODP Leg 119. Geol. Soc. Am. Abstr. Programs, 20:A252. (Abstract)

Berger, W. H., and Winterer, E. L., 1974. Plate stratigraphy and the fluctuating carbonate line. In Hsü, K. J., and Jenkyns, H. C. (Eds.), Pelagic Sediments on Land and Under the Sea: Spec. Publ. Int. Assoc. Sedimentol., 1:11-48.

Berggren, W. A., Kent, D. V., and Flynn, J. J., 1985. Jurassic to Paleogene: part 2. Paleogene geochronology and chronostratigraphy. In Snelling, N. J. (Ed.), The Chronology of the Geological Record: Geol. Soc. London Mem., 10:141-195.

Berggren, W. A., and Miller, K. G., in press. Cenozoic bathyal and abyssal benthic foraminiferal zonations. Micropaleontology.

Boersma, A., and Premoli-Silva, I., 1983. Paleocene planktonic foraminiferal biogeography and the paleoceanography of the Atlantic Ocean. Micropaleontology, 29:355-381.

Boersma, A., Shackleton, N. J., Hall, M., and Given, O., 1979. Carbon and oxygen isotope records at DSDP Site 384 (North 
Atlantic) and some Paleocene paleotemperatures and carbon isotope variations in the Atlantic Ocean. In Tucholke, B. E., Vogt, P. R., et al., Init. Repts. DSDP, 43: Washington (U.S. Govt. Printing Office), 695-717.

Braga, G., de Biase, R., Grunig, A., and Proto Decima, F., 1975. Foraminiferi bentonici del Paleocene ed Eocene della Sezione di Passagno. Schweiz. Palaontol. Abh., 97:85-111.

Brass, G. W., Southam, J. R., and Peterson, W. H., 1982. Warm saline bottom water in the ancient ocean. Nature, 296:620-623.

Ciesielski, P. F., Kristoffersen, Y., et al., 1988. Proc. ODP, Init. Repts., 114: College Station, TX (Ocean Drilling Program).

Corliss, B. H., and Keigwin, L. D., 1986. Eocene-Oligocene paleoceanography. In Hsü, K. J. (Ed.), Mesozoic and Cenozoic Oceans. Am. Geophys. Union Geodyn. Ser., 15:101-118.

Graham, D. W., Corliss, B. H., Bender, M. L., and Keigwin, L. D., Jr., 1981. Carbon and oxygen isotopic disequilibria of recent deep-sea benthic foraminifera. Mar. Micropaleontol., 6:483-497.

Hsü, K. J., McKenzie, J. A., and Weissert, H. J., 1985. Cenozoic carbon-isotope record in South Atlantic sediments. In Hsü, K. J., and Weissert, H. J. (Eds.), South Atlantic Paleoceanography: Oxford (Oxford Press), 189-196.

Katz, M. E., and Miller, K. G., 1988. Paleocene to Eocene benthic foraminiferal turnover, Atlantic sector Southern Ocean. Geol. Soc. Am. Abstr. Programs, 20:A251. (Abstract)

Keigwin, L. D., and Corliss, B. H., 1986. Stable isotopes in late middle Eocene to Oligocene forams. Geol. Soc. Am. Bull., 97:335345.

Kennett, J. P., and Stott, L. D., 1988. Antarctic Paleogene oxygen isotopic and climatic history, Maud Rise, Weddell Sea. Geol. Soc. Am. Abstr. Programs, 20:A251. (Abstract)

1990. Proteus and Proto-Oceanus: ancestral Paleogene oceans as revealed from Antarctic stable isotopic results; ODP Leg 113. In Barker, P., Kennett, J. P., et al., Proc. ODP, Sci. Results, 113: College Station, TX (Ocean Drilling Program), $865-880$.

Lohmann, G. P., 1980. PATS-1, a Package of Programs for the Analysis of Marine Micropaleontological Data on the VAX 11/780 Computer. Woods Hole Oceanogr. Inst. Tech. Rept., WHOI-8027.

Miller, K. G., and Curry, W. B., 1982. Eocene to Oligocene benthic foraminiferal isotopic record in the Bay of Biscay. Nature, 296:347-350.

Miller, K. G., Curry, W. B., and Ostermann, D. R., 1985. Late Paleogene (Eocene to Oligocene) benthic foraminiferal oceanography of the Goban Spur region, Deep Sea Drilling Project Leg 80. In de Graciansky, P. C., Poag, C. W., et al., Init. Repts. DSDP, 80: Washington (U.S. Govt. Printing Office), 505-538.

Miller, K. G., and Fairbanks, R. G., 1985. Oligocene to Miocene carbon isotope cycles and abyssal circulation changes. In Sundquist, E. J., and Broecker, W. S. (Eds.), The Carbon Cycle and Atmospheric $\mathrm{CO}_{2}$ : Natural Variations Archean to Present. Am. Geophys. Union Geophys. Monogr., 32:469-486.

Miller, K. G., Fairbanks, R. G., and Mountain, G. S., 1987a. Tertiary oxygen isotope synthesis, sea-level history, and continental margin erosion. Paleoceanography, 1:1-19.

Miller, K. G., Fairbanks, R. G., and Thomas, E., 1987b. Benthic foraminiferal carbon isotopic records and the development of abyssal circulation in the eastern North Atlantic. In Ruddiman, W. F., Kidd, R. B., and Thomas, E., et al., Init. Repts. DSDP, 94: Washington (U.S. Govt. Printing Office), 981-996.

Miller, K. G., Feigenson, M. D., Kent, D. V., and Olsson, R. K., 1988. Upper Eocene to Oligocene isotope $\left({ }^{87} \mathrm{Sr}{ }^{86} \mathrm{Sr}, \delta^{18} \mathrm{O}, \delta^{13} \mathrm{C}\right)$ standard section, Deep Sea Drilling Project Site 522. Paleoceanography, 3:223-233.

Miller, K. G., Janecek, T. R., Katz, M. E., and Keil, D. K., 1987c. Abyssal circulation and benthic foraminiferal changes near the Paleocene/Eocene boundary. Paleoceanography, 2:741-761.

Miller, K. G., and Katz, M. E., 1987. Oligocene to Miocene benthic foraminiferal and abyssal circulation changes in the North Atlantic. Micropaleontology, 33:97-149.

Morkhoven, F.P.C.M. van, Berggren, W. A., and Edwards, A. S., 1986. Cenozoic Cosmopolitan Deep-Water Benthic Foraminifera. Mem. Cent. Rech. Explor. Prod. Elf Aquitaine, 11.
Muza, J. P., Williams, D. F., and Wise, S. W., Jr., 1983. Paleogene oxygen record for Deep Sea Drilling Project Sites 511 and 512, subantarctic South Atlantic Ocean: paleotemperatures, paleoceanographic changes, and the Eocene/Oligocene boundary event. In Ludwig, W. J., Krasheninnikov, V. A., et al., Init. Repts. DSDP, 71: Washington (U.S. Govt. Printing Office), 409-422.

Oberhansli, H., McKenzie, J. A., Toumarkine, M., and Weissert, H., 1984. A paleoclimatic and paleoceanographic record of the Paleogene in the central South Atlantic (Leg 73, Sites 522, 523, and 524). In Hsü, K. J., LaBrecque, J. L., et al., Init. Repts. DSDP, 73: Washington (U.S. Govt. Printing Office), 737-747.

Oberhansli, H., and Toumarkine, M., 1985. The Paleogene oxygen and carbon isotope history of Sites 522, 523, and 524 from the central South Atlantic. In Hsü, K. J., and Weissert, H. J. (Eds.), South Atlantic Paleoceanography: Oxford (Oxford Press), 124147.

O'Neil, J. R., Clayton, R. N., and Mateda, T. K., 1969. Oxygen isotope fractionation in divalent metal carbonates. J. Chem. Phys., 51:5547-5558.

Savin, S. M., Douglas, R. G., and Stehli, F. G., 1975. Tertiary marine paleotemperatures. Geol. Soc. Am. Bull., 86:1499-1510.

Schnitker, D., 1979. Cenozoic deep water benthic foraminifera, Bay of Biscay. In Montadert, L., Roberts, D. G., et al., Init. Repts. DSDP, 48: Washington (U.S. Govt. Printing Office), 377-413.

Sclater, J. G., Anderson, R. N., and Bell, M. L., 1971. Elevation of ridges and evolution of the central eastern Pacific. J. Geophys., 76:7888-7915.

Shackleton, N. J., 1987. The carbon isotope record of the Cenozoic: history of organic carbon burial and of oxygen in the ocean and atmosphere. In Brooks, J., and Fleet, A. J. (Eds.), Marine Petroleum Source Rocks. Geol. Soc. Spec. Publ. London, 26:423434.

Shackleton, N. J., Corfield, R. M., and Hall, M. A., 1985a. Stable isotope data and the ontogeny of Paleocene planktonic foraminifera. J. Foraminiferal Res., 15:321-336.

Shackleton, N. J., and Hall, M. A., 1984. Carbon isotope data from Leg 74 sediments. In Moore, T. C., Jr., Rabinowitz, P. D., et al., Init. Repts. DSDP, 74: Washington (U.S. Govt. Printing Office), 613-619.

Shackleton, N. J., Hall, M. A., and Bleil, U., 1985b. Carbon isotope stratigraphy, 577. In Heath, G. R., Burckle, L. H., et al., Init. Repts. DSDP, 86: Washington (U.S. Govt. Printing Office), 503511.

Shackleton, N. J., Hall, M. A., and Boersma, A., 1984. Oxygen and carbon isotope data from Leg 74 foraminifers. In Moore, T. C., Jr., Rabinowitz, P. D., et al., Init. Repts. DSDP, 74: Washington (U.S. Govt. Printing Office), 599-612.

Shackleton, N. J., and Kennett, J. P., 1975. Paleotemperature history of the Cenozoic and the initiation of Antarctic glaciation: oxygen and carbon isotope analyses in DSDP Sites 277,279 and 281. In Kennett, J. P., Houtz, R. E., et al., Init. Repts. DSDP, 29: Washington (U.S. Govt. Printing Office), 743-755.

Shipboard Scientific Party, 1974. Site 245. In Simpson, E.S.W., Schlich, R., et al., Init. Repts. DSDP, 25: Washington (U.S. Govt. Printing Office), 187-236.

1988a. Site 698. In Ciesielski, P. F., Kristoffersen, Y., et al., Proc. ODP, Init. Repts., 114: College Station, TX (Ocean Drilling Program), 87-150.

1988b. Site 699. In Ciesielski, P. F., Kristoffersen, Y., et al,, Proc. ODP, Init. Repts., 114: College Station, TX (Ocean Drilling Program), 151-254.

1988c. Site 700. In Ciesielski, P. F., Kristoffersen, Y., et al., Proc. ODP, Init. Repts., 114: College Station, TX (Ocean Drilling Program), 255-362. 1988d. Site 702. In Ciesielski, P. F., Kristoffersen, Y., et al., Proc. ODP, Init. Repts., 114: College Station, TX (Ocean Drilling Program), 483-548.

Thomas, E., 1988. The Paleogene deep-sea environment at high latitudes. Geol. Soc. Am. Abstr. Programs, 20:A252. (Abstract) 1990. Late Cretaceous through Neogene deep-sea benthic foraminifers (Maud Rise, Weddell Sea, Antarctica). In Barker, P., Kennett, J. P., et al., Proc. ODP, Sci. Results, 113: College Station, TX (Ocean Drilling Program), 571-594. 
Thomas, E, in press. Mass extinctions in the deep sea. In Global Catastrophes in Earth History. Geol. Soc. Am. Spec. Publ.

Tjalsma, R. C., 1976. Cenozoic foraminifera from the South Atlantic, DSDP Leg 36. In Barker, P. F., Dalziel, I.W.D., et al., Init. Repts. DSDP, 36: Washington (U.S. Govt. Printing Office), 493-518.

Tjalsma, R. C., and Lohmann, G. P., 1983. Paleocene-Eocene bathyal and abyssal benthic foraminifera from the Atlantic Ocean. Micropaleontol. Spec. Publ: 4:1-90.

Vergnaud-Grazzini, C., and Oberhansli, H., 1986. Isotopic events at the Eocene/Oligocene transition: a review. In Pomerol, C., and Premoli Silva, I. (Eds.), Terminal Eocene Events: Amsterdam (Elsevier), 311-329.

Vergnaud-Grazzini, C., Pierre, C., and Letolle, R., 1978. Paleoenvironment of the northeast Atlantic during the Cenozoic: oxygen and carbon isotope analyses of DSDP Sites 398, 400A, and 401. Oceanol. Acta, 11:381-390.
Vincent, E., Gibson, J. M., and Brun, L., 1974. Paleocene and early Eocene microfacies, benthonic foraminifera and paleobathymetry of Deep Sea Drilling Project Sites 236 and 237, western Indian Ocean. In Fisher, R. L., Bunce, E. T., et al., Init. Repts. DSDP, 24: Washington (U.S. Govt. Printing Office), 859-885.

Wood, K. C., Miller, K. G., and Lohmann, K. P., 1985. Middle Eocene to Oligocene benthic foraminifera from the Oceanic Formation, Barbados. Micropaleontology, 31:181-196.

Date of initial receipt: 14 April 1989

Date of acceptance: 15 January 1990

Ms 114B-147 


\section{$\%$ S. beccariiformis}

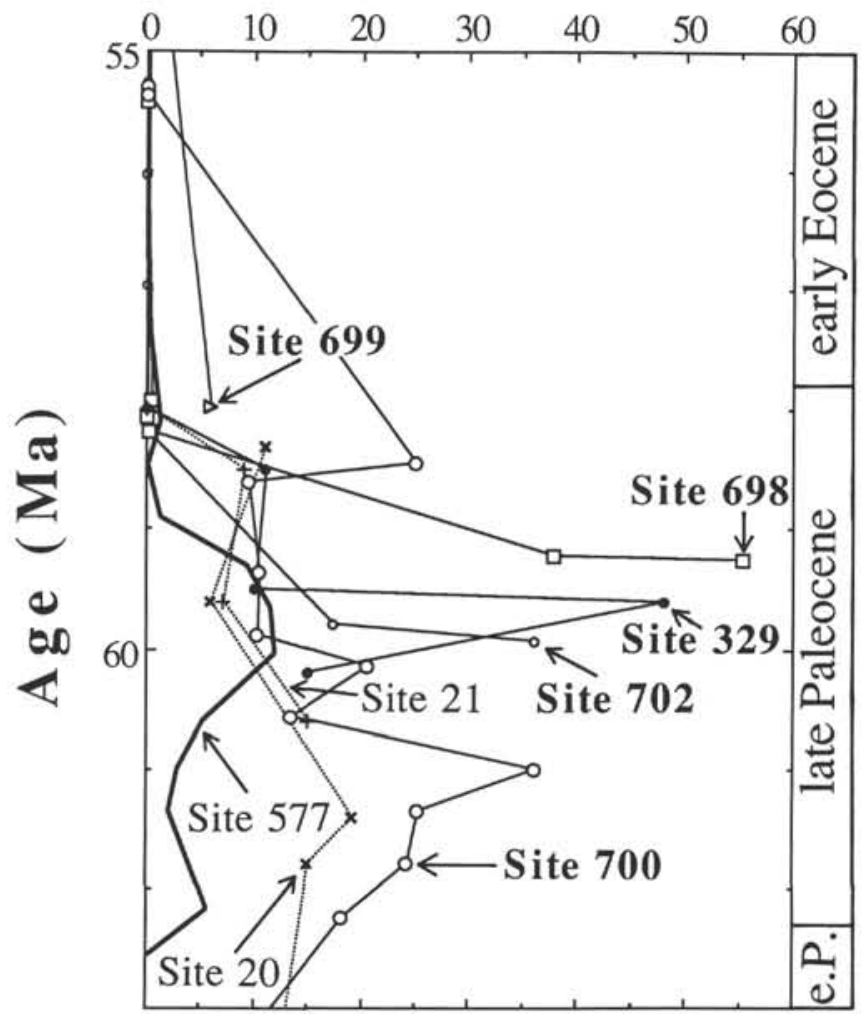

Figure 13. Relative abundance of Stensioina beccariiformis at selected locations in the Atlantic, Pacific, and Southern oceans. Leg 114 sites are indicated with open symbols and solid lines; DSDP Site 329 (solid line, solid circles) is a Southern Ocean site also (data after Tjalsma and Lohmann, 1983); Atlantic DSDP Sites 20 and 21 (data after Tjalsma and Lohmann, 1983) are indicated with dashed lines; Pacific DSDP Site 577 data (after Miller et al., 1987c) are indicated with a solid line (no points plotted). Note that percentages of this taxon are highest in the Southern Ocean region (Sites 329, 698, 699, 700 , and 702). 
$\delta^{18} \mathrm{O}$

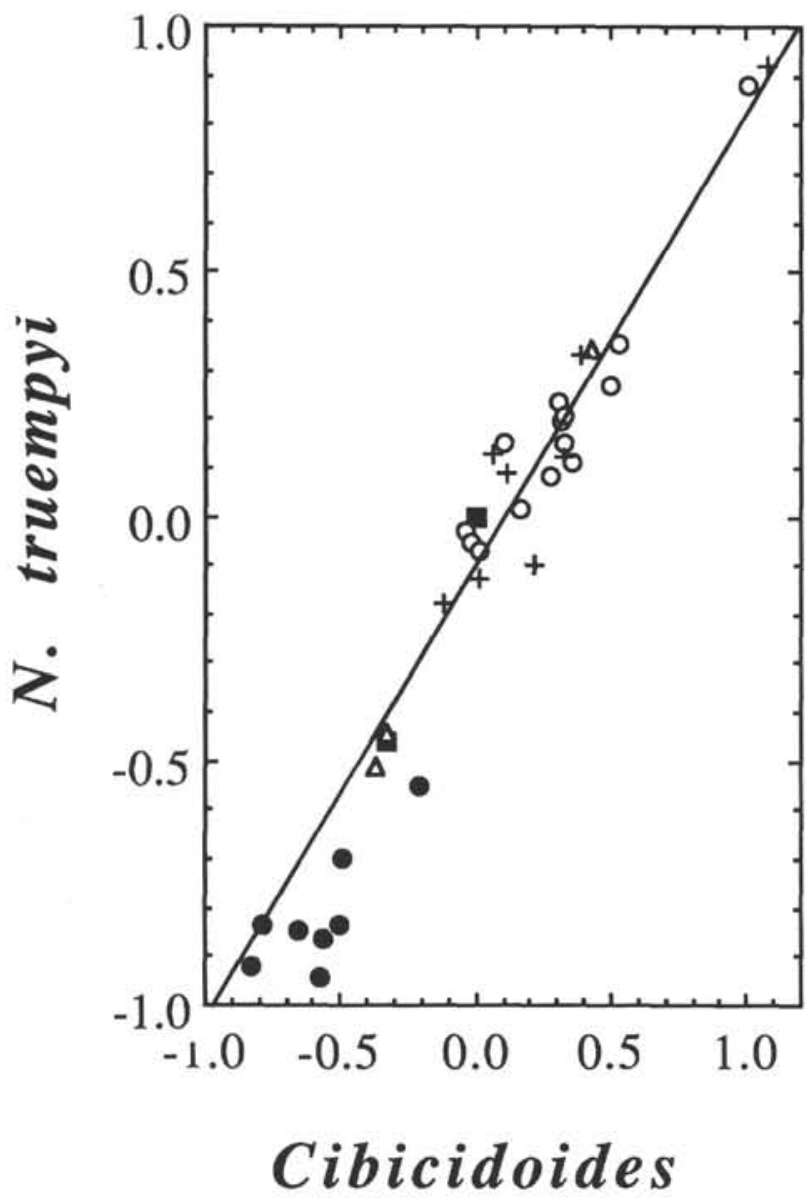

$\delta^{13} \mathrm{C}$

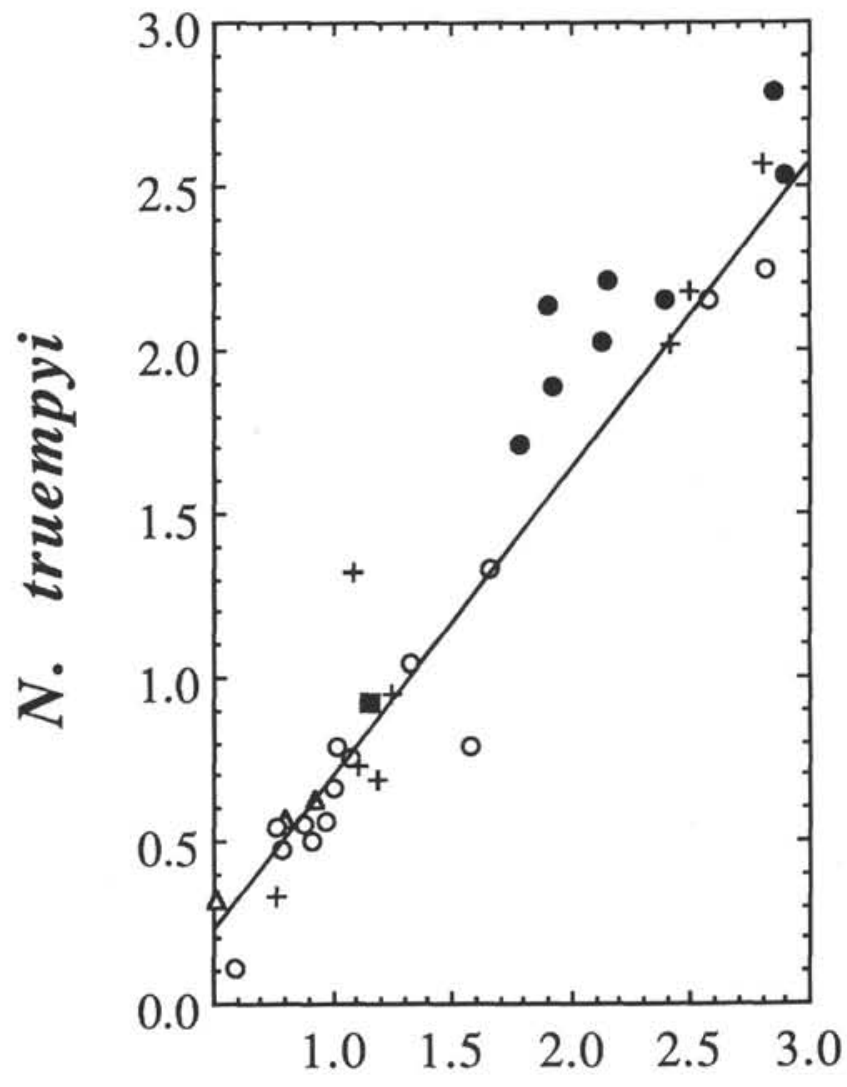

Cibicidoides

Figure 14. Paired Nuttallides truempyi and Cibicidoides spp. isotope analyses plotted against each other for Hole 702B (open circles), Hole 700B (squares), recrystallized samples from Hole 700B (solid circles; data not included in regression line), Site 384 (triangles), and data from Shackleton et al. (1984) (crosses). 
M. E. KATZ, K. G. MILLER

HOLE 702B

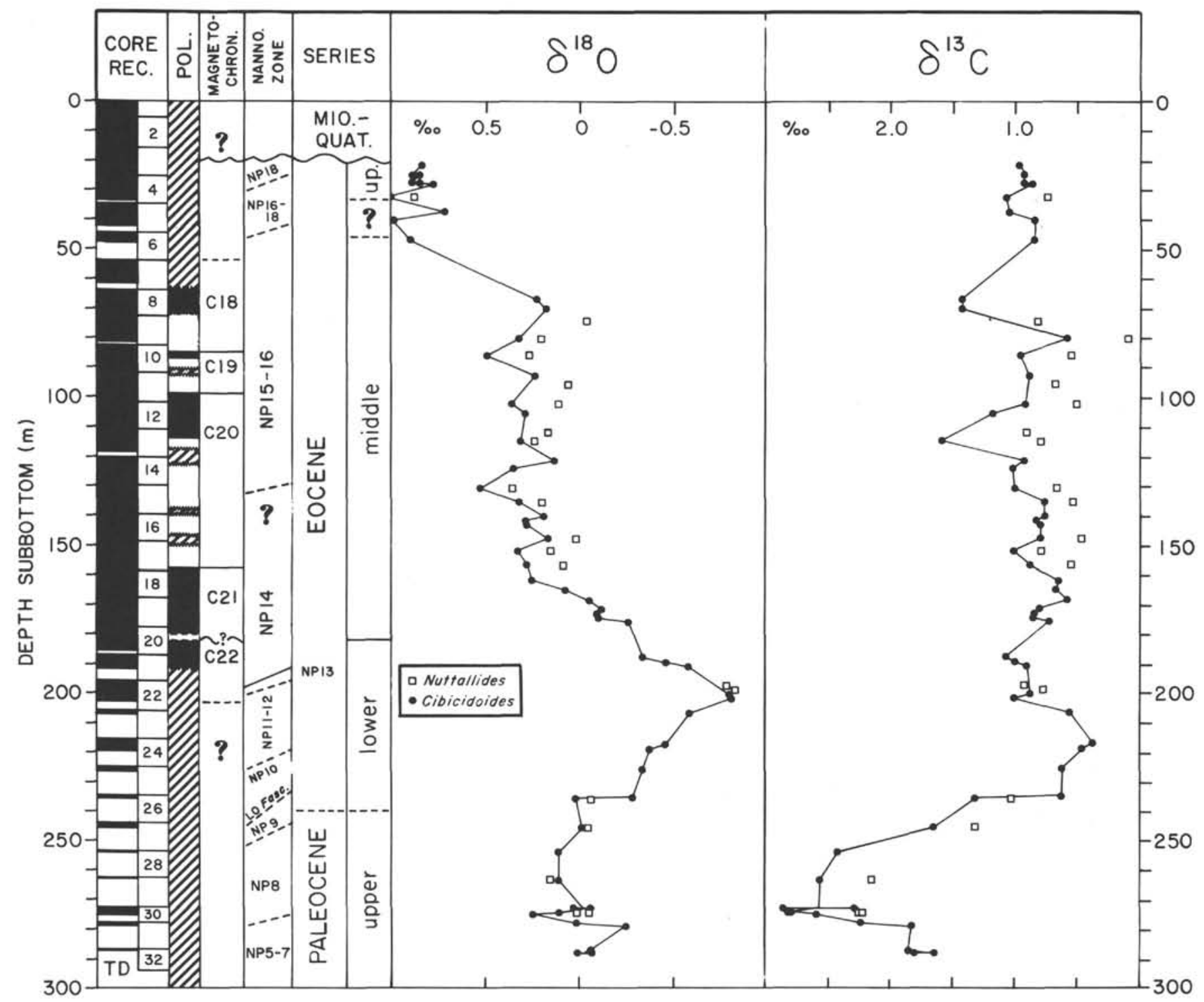

Figure 15. Oxygen and carbon isotope analyses of Cibicidoides spp. (solid circles) and Nuttallides truempyi (open squares) at Hole 702B (several analyses from Hole 702A are corrected to Hole 702B sub-bottom depths; Table 2). Columns as in Figure 6. The plotted duplicates are connected with a horizontal line. 


\section{HOLE 700B}

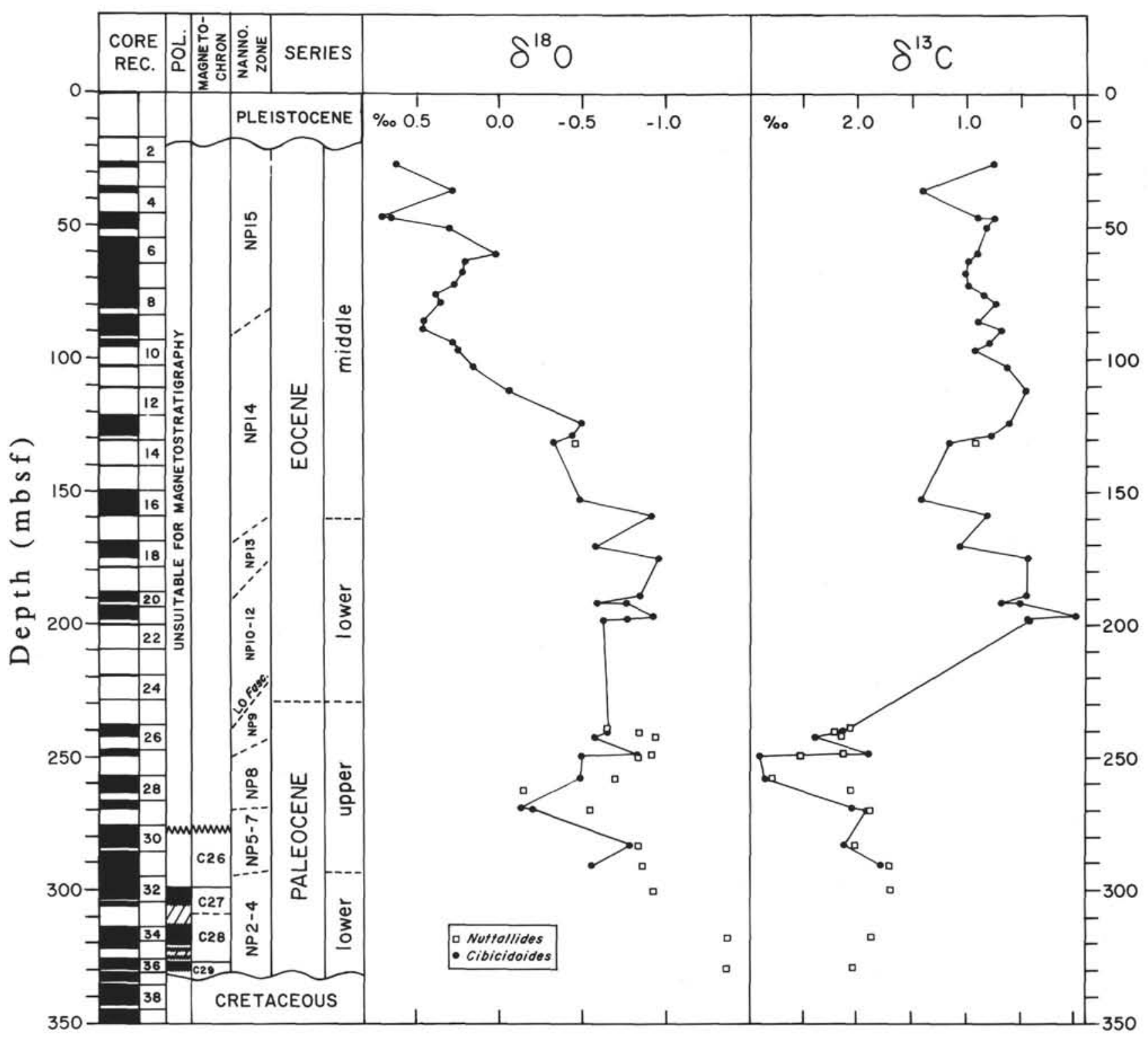

Figure 16. Oxygen and carbon isotope analyses of Cibicidoides spp. (solid circles) and Nuttallides truempyi (open squares) at Hole 700B. Note that specimens below approximately 220 mbsf were recrystallized and yielded unreliable results. Columns as in Figure 8 . 


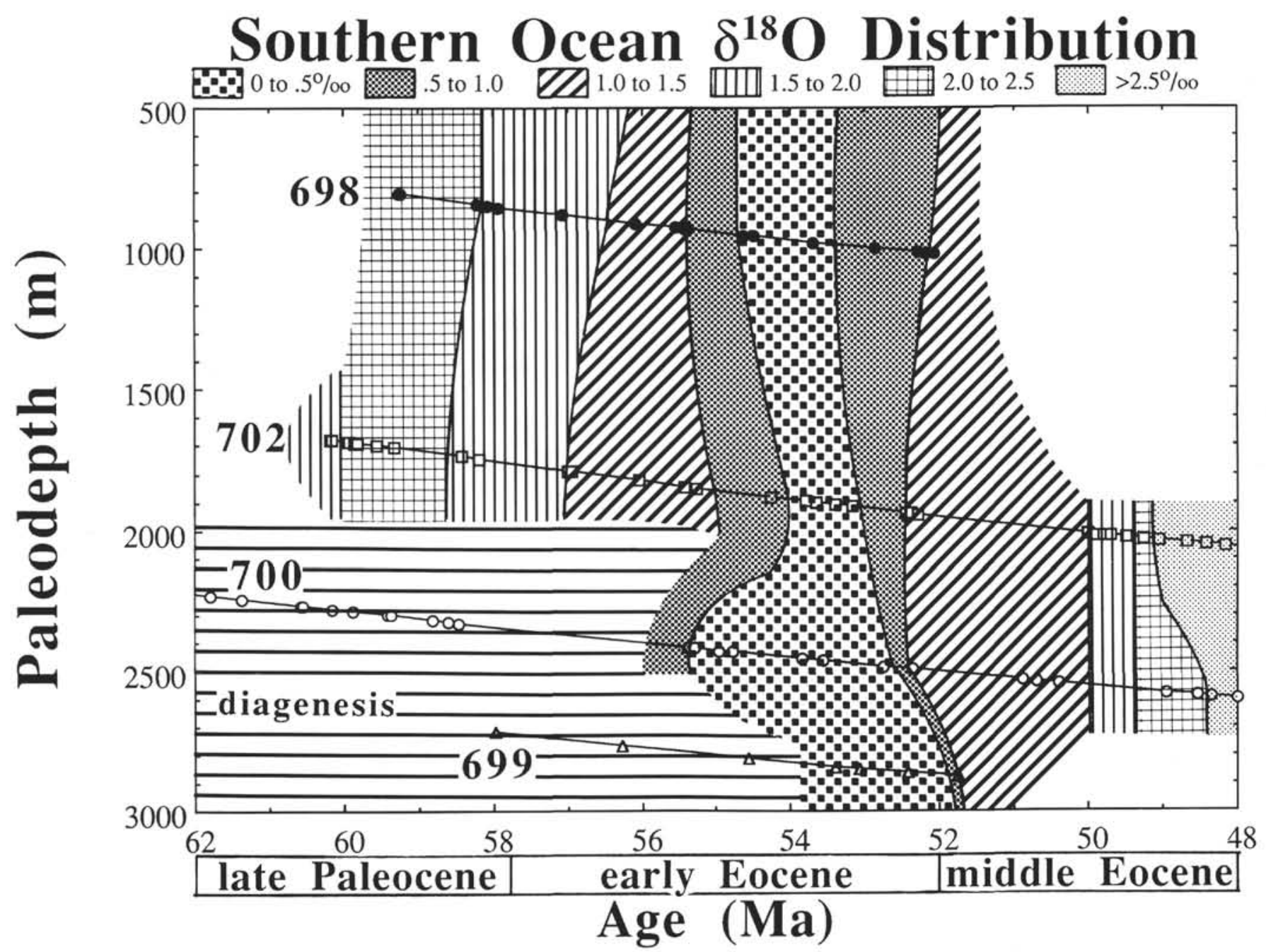

Figure 17. Age-paleodepth reconstruction for oxygen isotope data from Leg 114 sites. Only Cibicidoides spp. data were considered from Holes $698 \mathrm{~A}, 700 \mathrm{~B}$, and 702B; Hole 699A is based entirely upon Nuttallides truempyi data. The Paleocene section at Site 700 and the Paleocene to lowermost Eocene section at Site 699 are diagenetically altered and are not contoured (see text). 


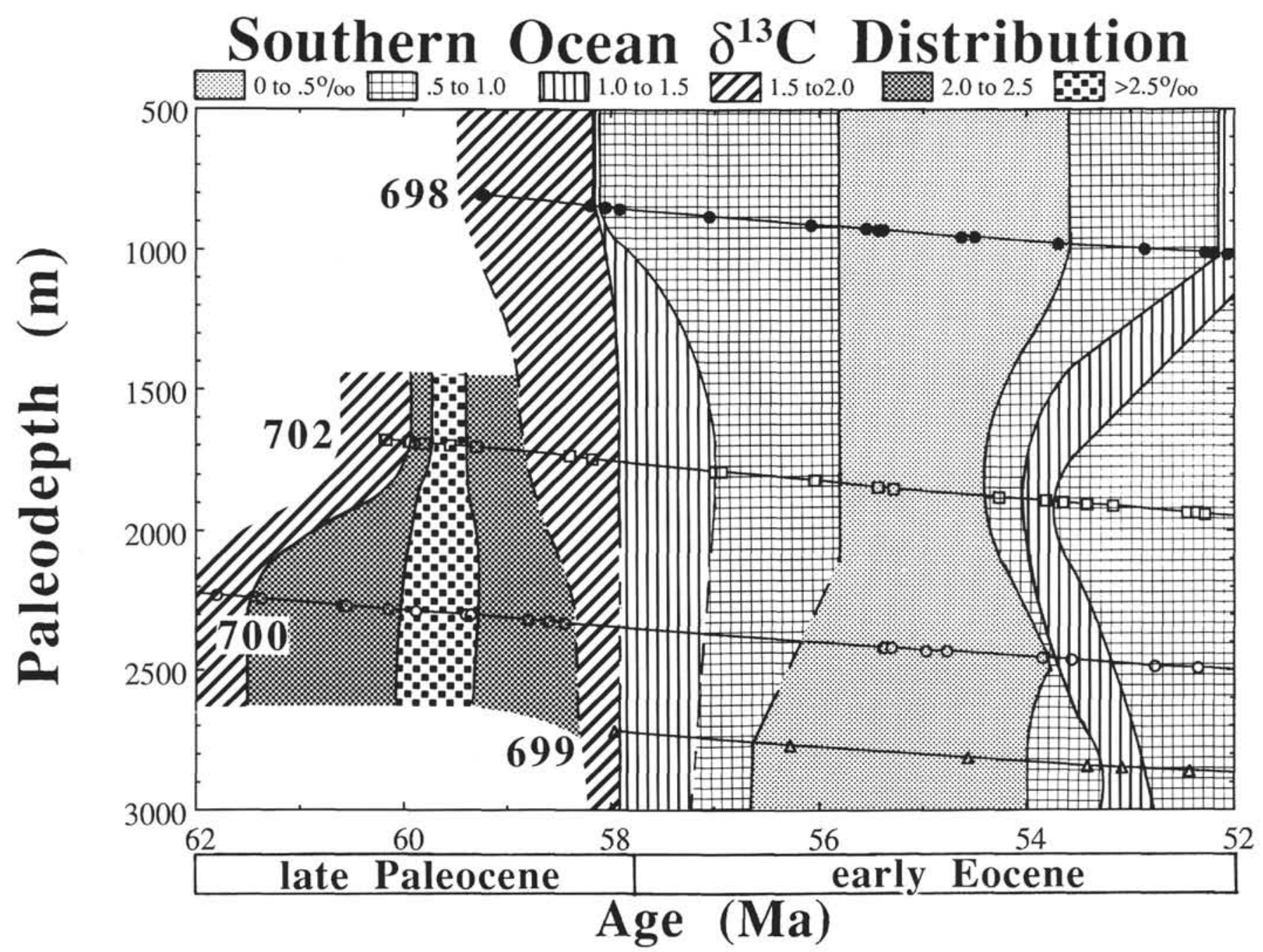

Figure 18. Age-paleodepth reconstruction for carbon isotope data from Leg 114 sites. Only Cibicidoides spp. data were considered from Holes 698A, 700B, and 702B; Hole 699A is based entirely upon Nuttallides truempyi data. 

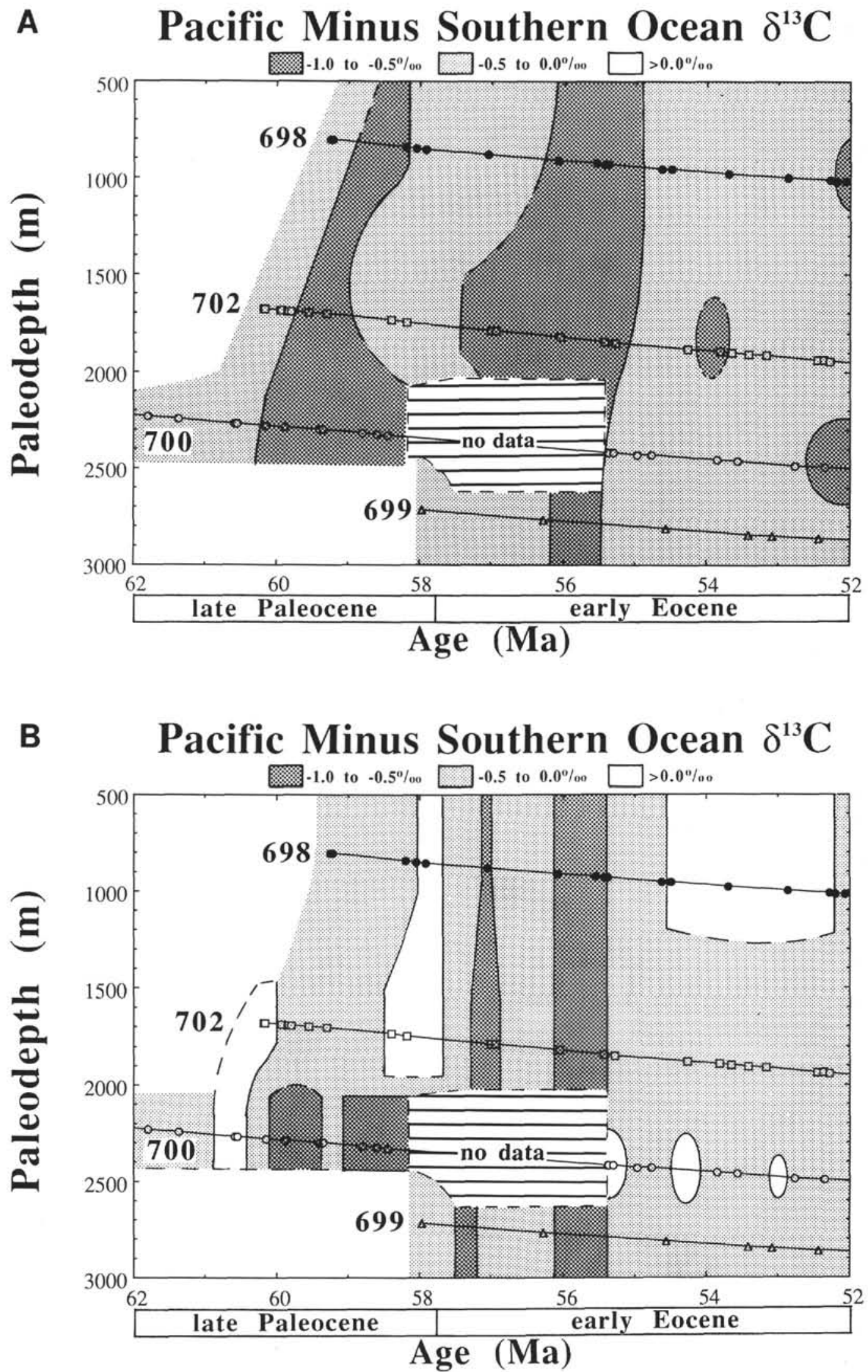

Figure 19. Age-paleodepth reconstruction for carbon isotope data from Leg 114 sites subtracted from Pacific Site 577 data values. Data were interpolated at $0.25-\mathrm{m}$.y. intervals, the difference determined, and contoured. A. Only Cibicidoides spp. data were considered from Holes 698A, 700B, and 702B; Hole 699A is based entirely upon Nuttallides truempyi data. B. Both Cibicidoides spp. and $N$. truempyi data from Leg 114 locations (Table 2). All $N$. truempyi data were corrected by subtracting $0.26 \%$. 


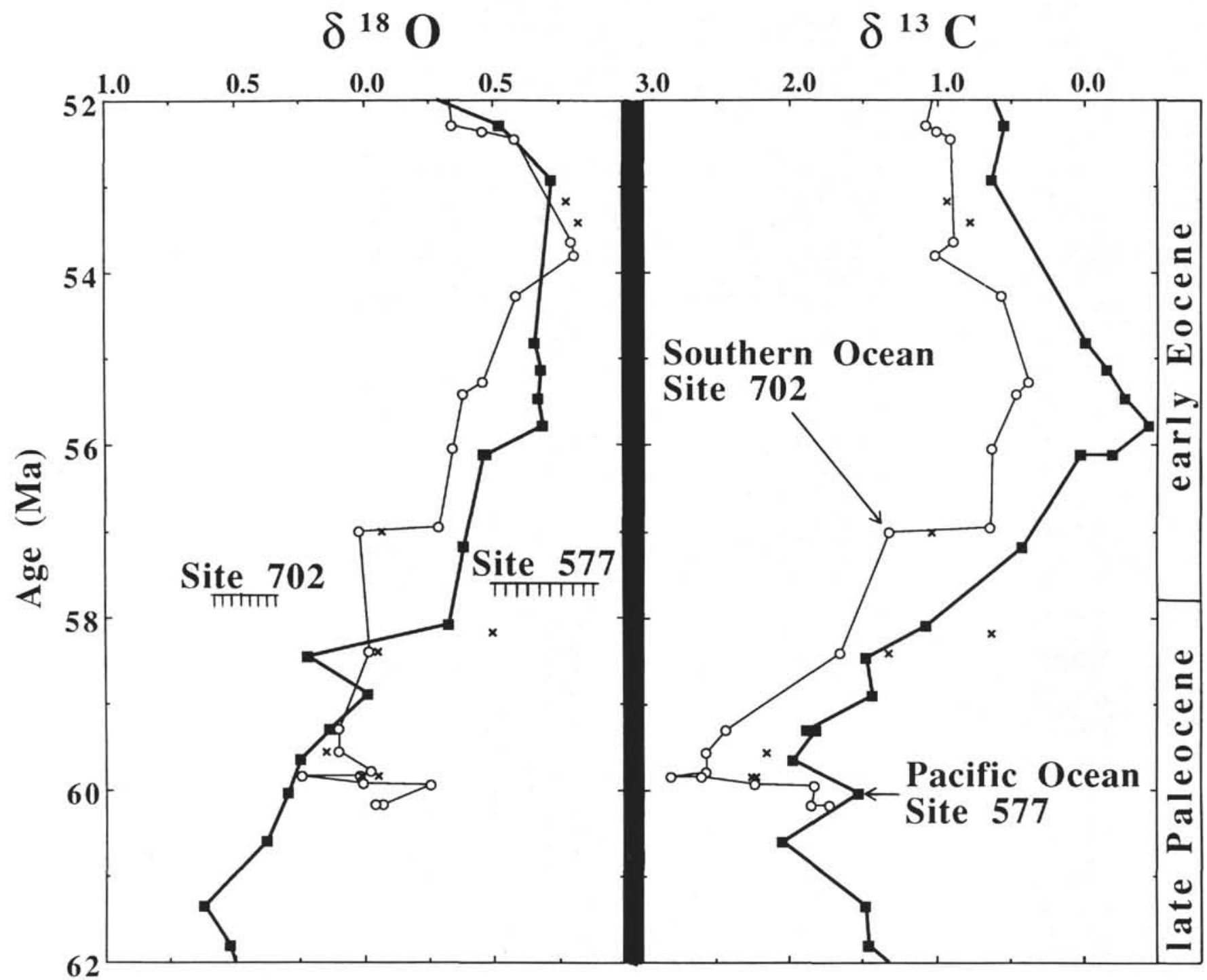

Figure 20. Comparison between Pacific Sites 577 (solid squares; Nuttallides truempyi data) with Southern Ocean Site 702 (open circles; Cibicidoides spp. data). No corrections were applied to data from either of these two sites. N. truempyi data from Sites 702 are shown as unconnected $\times$ 's. The benthic foraminiferal extinctions at Sites 577 and 702 are individually plotted between samples as T's. 


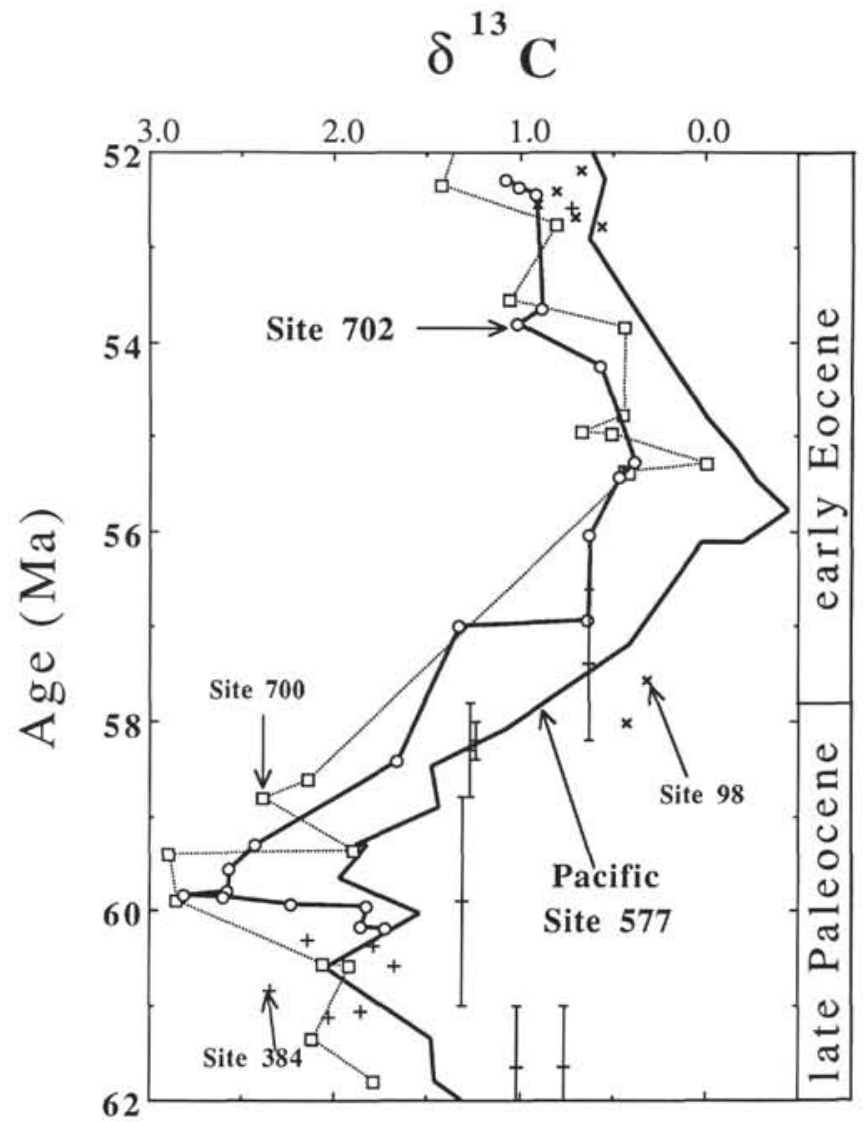

Figure 21. Comparison of carbon isotope records from Pacific Site 577 (solid line, no points; Nuttallides truempyi uncorrected data) with Southern Ocean Sites 700 (open squares, dotted line; Cibicidoides spp. data) and 702 (open circles, solid line; Cibicidoides spp. data) and western Atlantic Sites 98 (×'s; Cibicidoides spp. data) and 384 (crosses; Cibicidoides and N. truempyi data). Also shown are data from western Atlantic Sites 20, 21, 144, 356, and 357 ( N. truempyi, uncorrected) plotted individually as horizontal lines with error bars, which represent the ages of the assigned planktonic foraminiferal zones. 


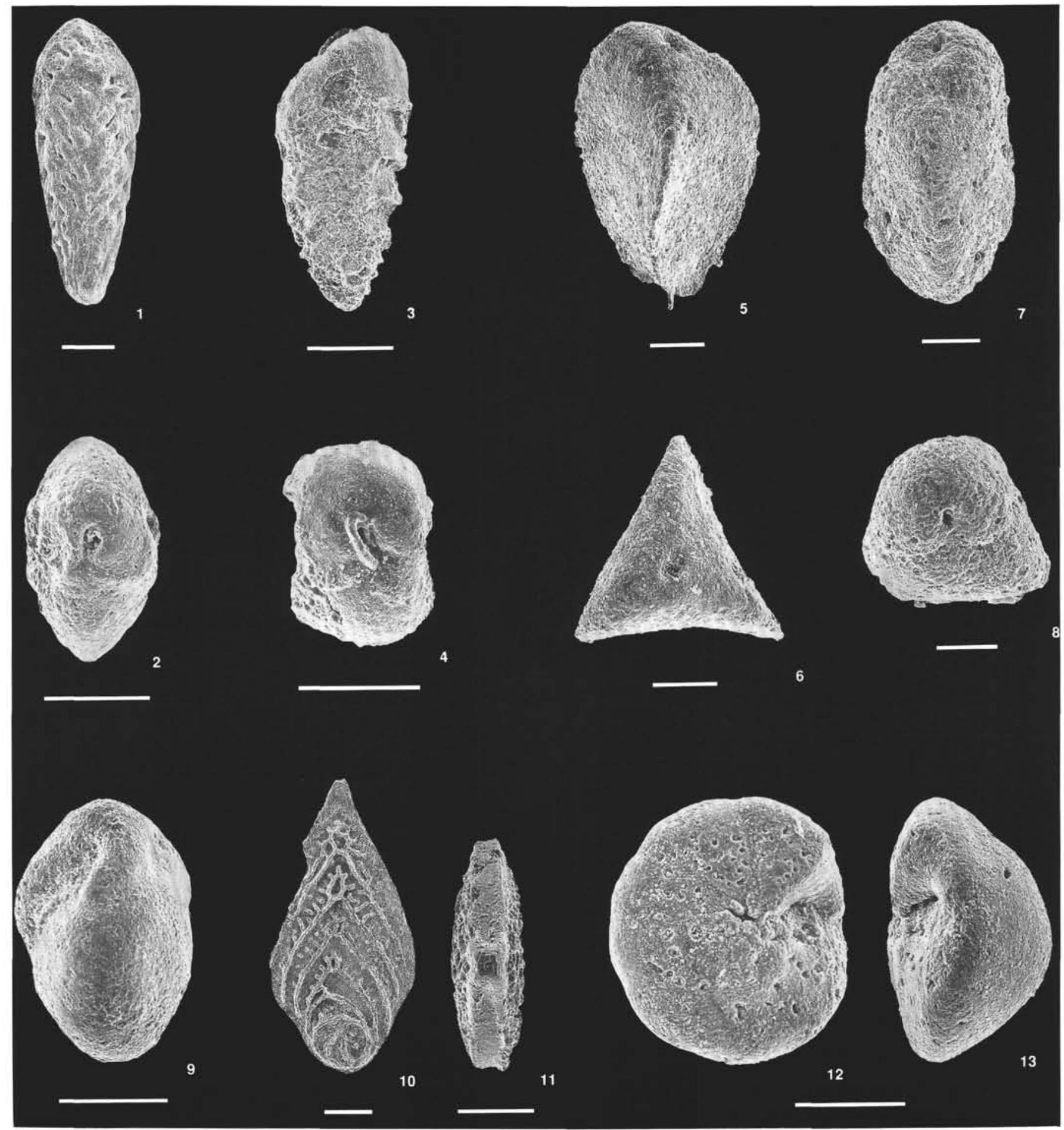

Plate 1. Taxa characteristic of (but not restricted to) the Paleocene. Scale bar $=100 \mu \mathrm{m} .1$, 2. Bolivinoides delicatulus Cushman, Sample 114-699A-54X-2, 22-24 cm. 3, 4. Tappanina selmensis (Cushman), Sample 114-698A-9R-2, 16-20 cm. 5, 6. Tritaxia havanensis (Cushman and Bermudez), Sample 114-699A-54X-2, 22-24 cm. 7, 8. Tritaxia paleocenica Tjalsma and Lohmann, Sample 114-699A-54X-2, 22-24 cm. 9. Alabamina creta (Finlay), Sample 114-700B-32R-4, 55-59 cm. 10, 11. Neoflabellina semireticulata (Cushman and Jarvis), Sample 114-700B26R-2, 60-64 cm. 12, 13. Neoeponides hillebrandti Fisher, Sample 114-699A-54X-2, 22-24 cm. 


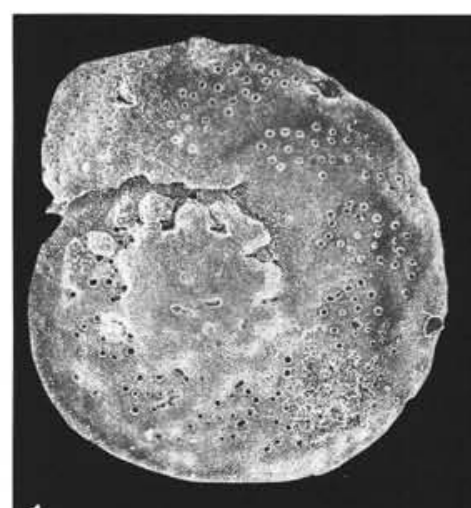

1

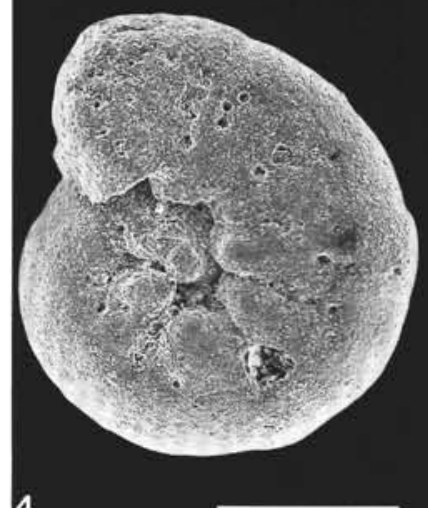

4

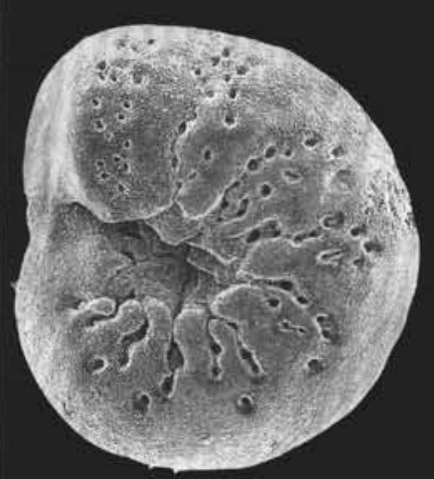

8

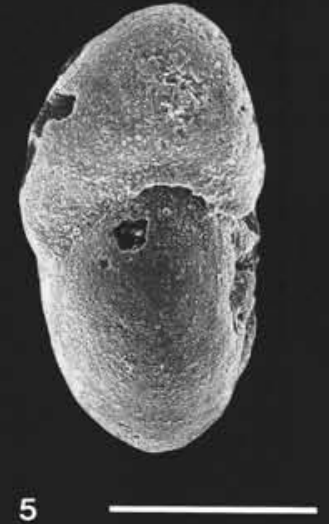

5

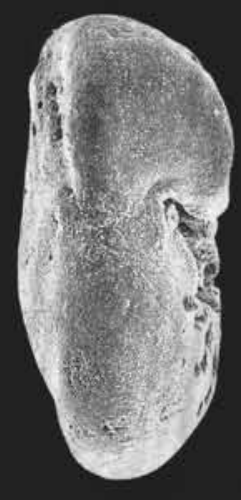

9

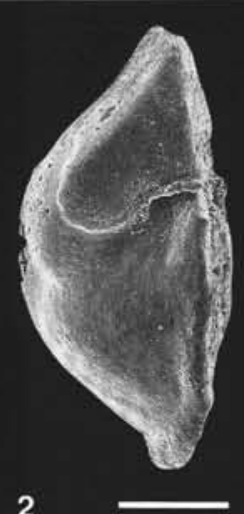

2

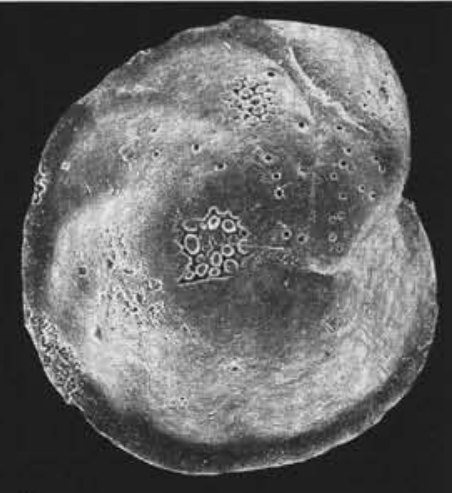

3

Plate 2. Taxa characteristic of (but not restricted to) the Paleocene. Scale bar $=100 \mu \mathrm{m}$. 1-3. Cibicidoides aff. subspiratus (Nuttall), Sample 114-698A-6R-1, 140-144 cm. 4, 5. Cibicidoides hyphalus (Fisher), Sample 114-702B-30X-1, 30-34 cm. 6, 7. Cibicidoides velascoensis (Cushman), Sample 114-700B-34R-3, 80-82 cm. 8, 9. Stensioina beccariiformis (White), Sample 114-702B-30R-1, 30-34 cm. 10, 11. Anomalinoides danicus (Brotzen), Sample 114-698A-10R-1, 144-148 cm. 


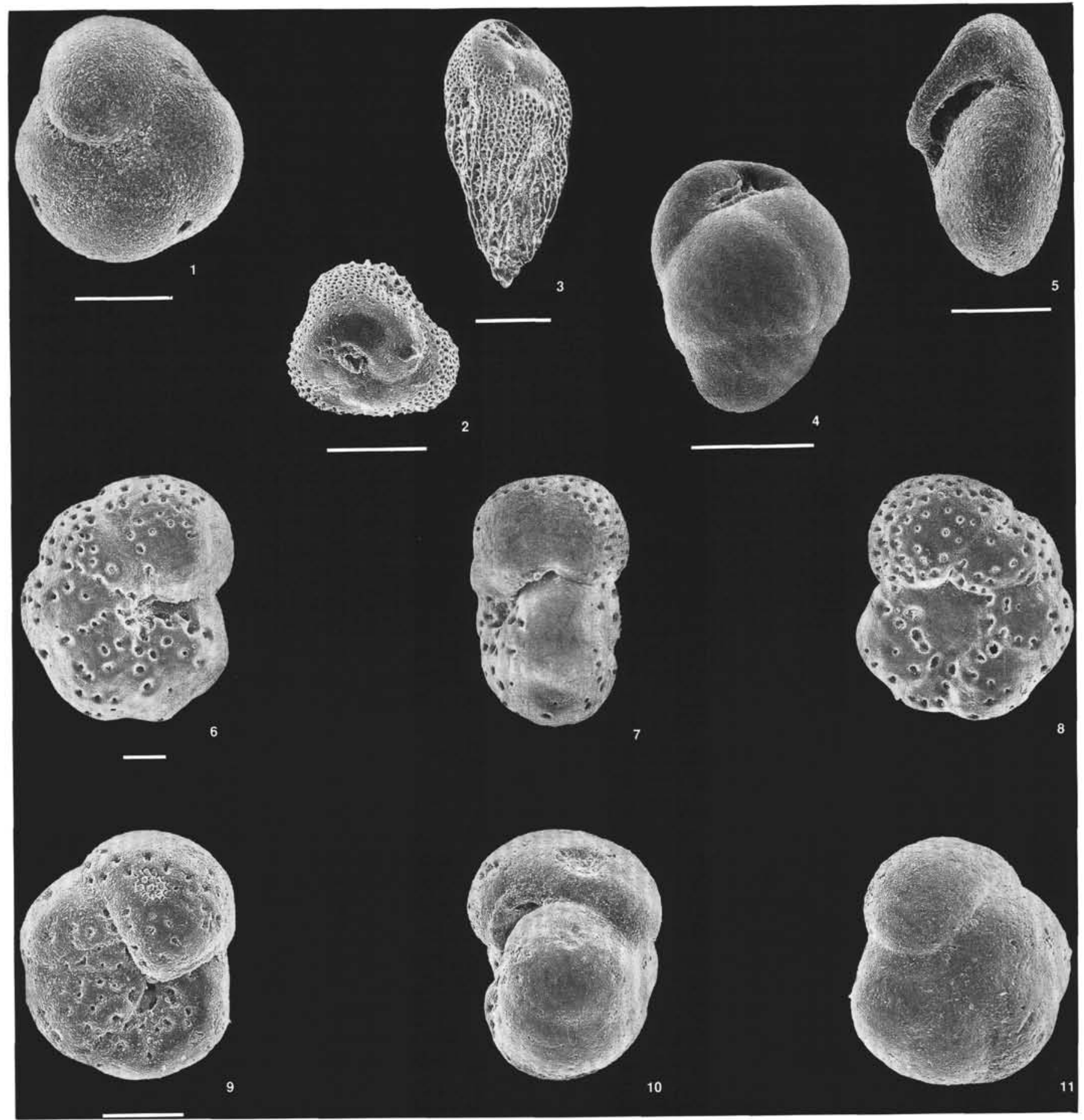

Plate 3. Taxa characteristic of (but not restricted to) the Eocene. Scale bar $=100 \mu \mathrm{m}$. 1. Abyssamina sp., Sample 114-700B-6R-6, 72-76 cm. 2, 3. Bulimina semicostata Nuttall, Sample 114-702B-18X-4, 20-24 cm. 4. Turrilina robertsi (Howe and Ellis), Sample 114-698A-6R-2, 32-36 cm. 5. Clinapertina sp., Sample 114-702B-23X-1, 49-53 cm. 6-8. Anomalinoides capitatus (Gumbel), Sample 114-702B-14X-3, 68-72 cm. 9-11. Anomalinoides semicribratus (Beckmann), Sample 114-702B-14X-3, 68-72 cm. 

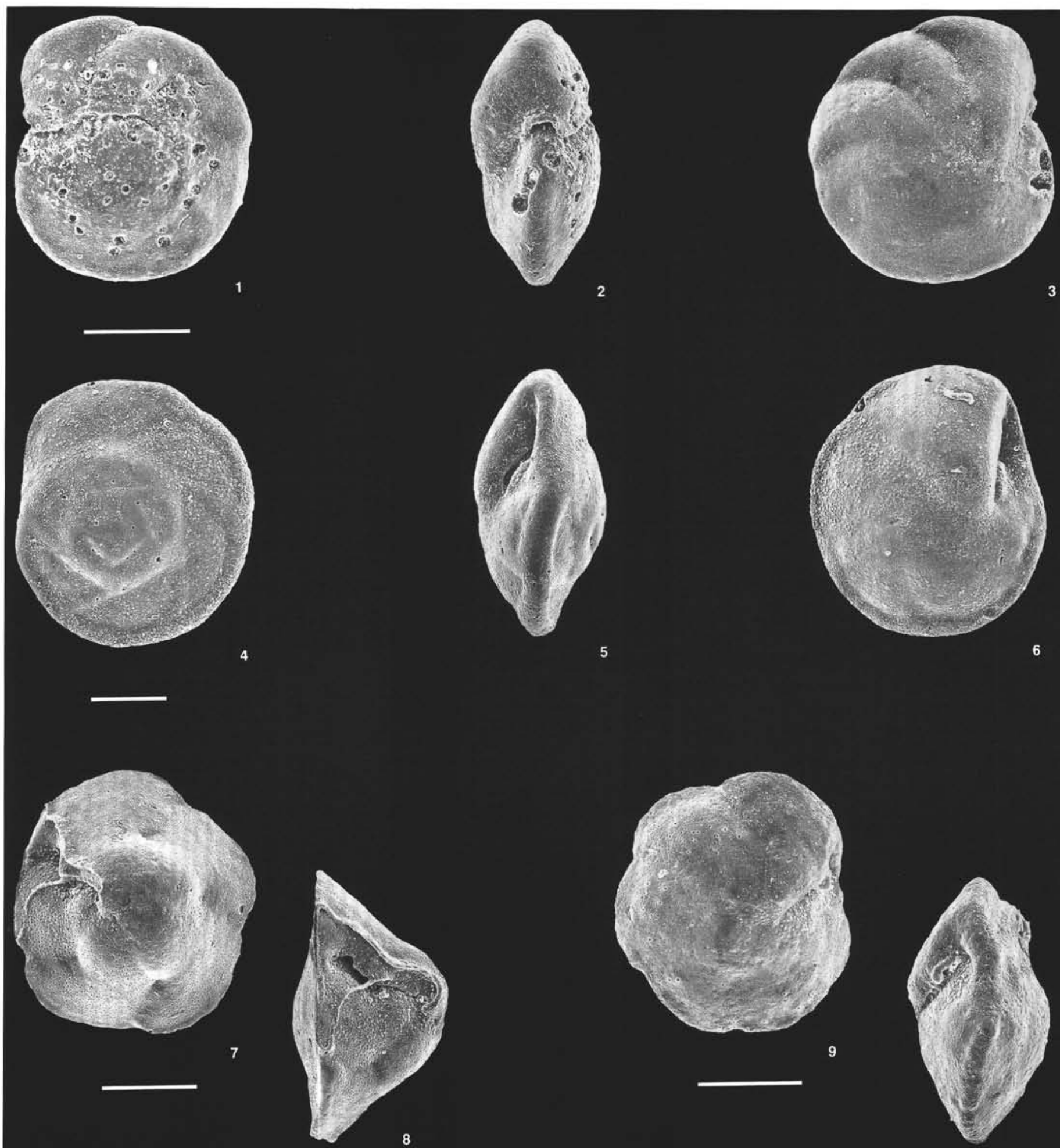

Plate 4. Taxa characteristic of (but not restricted to) the Eocene. Scale bar $=100 \mu \mathrm{m}$. 1-3. Cibicidoides praemundulus Berggren and Miller, Sample 114-702A-4H-2, 68-72 cm. 4-6. Alabamina dissonata (Cushman and Renz), Sample 114-700B-6R-6, 72-76 cm. 7, 8. Nuttallides truempyi (Nuttall), Sample 114-700B-6R-6, 72-76 cm. 9, 10. Nuttallides umbonifera (Cushman), Sample 114-702B-6X-2, 100-104 cm. 